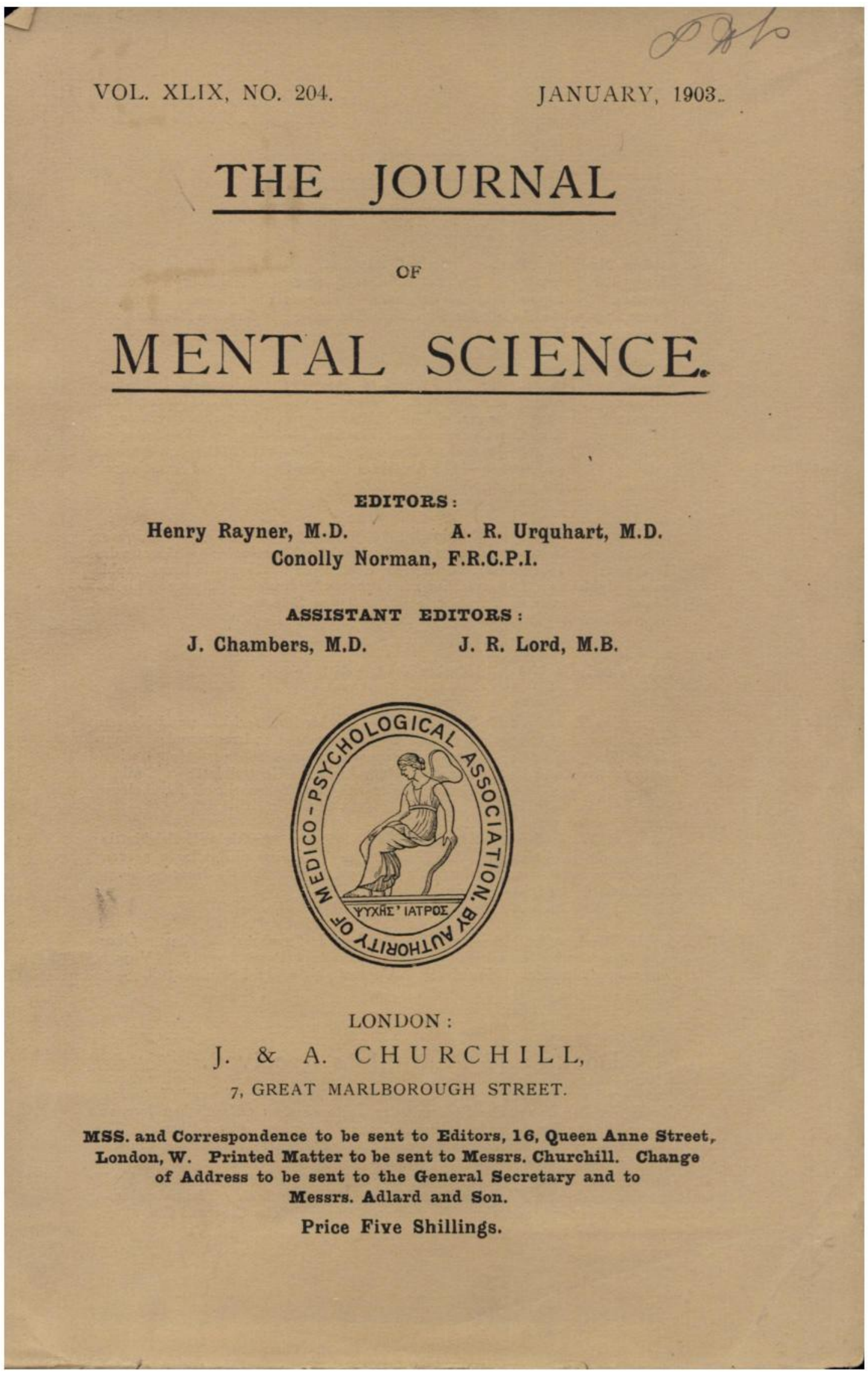




\section{JOURNAL OF MENTAL SCIENCE.}

\section{Exchange Journals.}

Dritioh.

Asylum News; Brain; British Medical Journal; Bristol MedicoChirurgical Journal; Dublin Medical Journal; Edinborgh Medical Journal; Glasgow Medical Journal; Hospital; Lancet; Mind; Proceedings of the Society for Psychical Research; Scottish Medical and Surgical Journal.

Amerioan.

Alienist and Neurologist; Journal of Insanity; Journal of Medical Sciences; Journal of Comparative Neurology; Journal of Mental and Nervous Diseases; Journal of Mental Pathology; Journal of Psychology; Medicine; Monthly Cyclopsedia of Practical Medicine; Psychological Review; Quarterly Journal of Inebriety; Reports of the Smithsonian Institute; Transactions of the American Medico-Psychological Association; Universal Medical Journal.

French.

Archives Anthropologie Criminelle; Annales Medica-Psychologiques; Annales des Sciences Psychiques; Archives de Neurologie; Gazette des Hopitaux; Journal de Médecine de Bordeaux; Nouvelle Iconographie de la Salpêtrière; Polybiblion; Progrès Médicale; Revue Philosophique; Revue de Psychiatrie; Revue Scientifique; Revue de j'Hypnotisme; Bulletin de l'Institut Psychique.

Delgian.

Bulletin de la Société de Médecine Mentale de Belgique.

arrman.

Algemeine Zeitschrift für Psychiatrie; Archiv für Psychiatrie und Nervenkrankheiten; Centralblatt für Anthropologie; Der Irrenfreund; Jahrbücher für Psychiatrie; Kraepelin's Psychologische Arbeiten; Monatsschrift für Psychiatrie und Neurologie; Neurologisches Centralblatt; Philosophische Studien; Psychiatrische Wochenschrift; Zeitschrift für Psychologie.

Italian.

Annali di Freniatria; Annali di Nevrologia; Archivio di Psichiatria; I) Manicomio Moderno; La Psichiatria ; Rivista di Patologia nervosa e mentale; Rivista Sperimentale di Freniatria; Rivista Neuropatologia Psichiatria ; Rivista di Psicologia.

Inarian.

Archiv Psychiatrii, Nevrologuii, i Soudebnoi Psychopatologuii; Obozrenie Psychiatrii, Nevrologuii, i Experimentalnoi Psychologuii; Voprosi Nervno-psychitscheckoi Medizini; Voprosi philosophit i psychologuii.

\section{Books and Pamphlets Recelved.}

An Essay on Laughter, Sully, L'Association des Idees, Dr. Bdouard Clapardde; La Valonte, Fr. Paulhan; Die Stellung der Xrzte an dem Offentlichen Irrenanstalten, Hugo Hoppe; Troubles de la Parole dans l'Epilepsie, Albert Vincent; The Rise of the Experimental Method in Oxford, Ctifford Allbutt; Das biomechanische Denken in der Medizin und in der Biologie, Moris Benedikt; Geschlecht und Krankheit, Mobius; The Mattison Method in Morphinism, F. B. Mattison; Der Achillessehnenreflex und seine klinische Bedeutung, Arthur won Sarbb; The Selection of Consumptive Cases for Sanatorium Treatment, Kelynack; The Care and Custody of the Convict and Criminal Insane, $H . E$. Allison. 


\title{
MEDICO-PSYCHOLOGICAL ASSOCIATION OF GREAT BRITAIN AND IRELAND.
}

\author{
THE COUNCIL AND OFFICERS, 1902-3.
}

PRBSIDRNT. JOSEPH WIGLESWORTH, M.D.

PRESIDENT ELBCT.-ERNEST W. W HITE, M.B.

EX-PRESIDENT.-OSCAR T. WOODS, M.D.

TRRASURRR.-H. HAYES NEWINGTON, F.R.C.P.Ed.

EDITORS OF JOURNAI. $\left\{\begin{array}{l}\text { HENRY RAYNER, MI.D. } \\ \text { A. R. URQUHART, MI.D. }\end{array}\right.$

CONOLLY NORAAN, F.R.C.P.I.

AsSISTANT RDITORS. S JAMIES CHAMBERS. ML.D.

(Not Members of Council.) JOHN R. LORD, M.B.

AUDTTORS. $\left\{\begin{array}{l}\text { JAMES M. MOODY, M.R.C.S. } \\ \text { E. B. WHITCOMBE, M.R.C.S. }\end{array}\right.$

DIVISIONAL BECRETARY FOR BUUTH -EASTBRN DIVIBION.-A. N. BOYCOTT, M.D. DIVIBIONAL SECRETARY POR BOUTH - WESTERN DIVISION. - P. W. MACDONALD, M.D. DIVIBIONAL BBCRETAKY FOR NORTHBHN AND MIDLAND DIVISION.

C. K. HITCHCOCK, M.D.

DIVIBIONAL SEcretaRY FOR 8COTLAND.-LEWIS C. BRUCE, M.B.

DIVIBIONAL SRCRETARY FOR IRELAND.-W. R. DAWSON, M.D.

GRNeral SECRetarY.-ROBERT JONES, M.D., B.S., F.R.C.S.

BbCretary of edecational committer.-C. A. MERCIER, M.B. (appointed by Educational Committee, but with seat on Council).

REGISTRAR. -ALFRED MILLER, M.B.

MEMBRRS OF COUNCIL.

C. H. BOND, M.D.

1900. R. L. RUTHERFORD, M.D. 1901.

J. G. HAVELOCK, M.D. , J. BEVERIDGE SPENCE, M.D. "

F. P. HEARDER, M.D.

H. GARDINER HILL,

ALFBED MILLER, M.B.

ALFRED MILLER, M.B.

T. S. ADAIR, M.B.

THEO. B. HYSLOP, M.D.

H. A. KIDD, M.R.C.S.

$\because \quad$ A. R. TORNBULY

A. R. TURNBUL.L.M.B.
R. C. STEWAR'T, M.B.

F. W. MOTT, M.D.

" F. W. MOTT

, A. D. O’C. FINEGAN, L.R.C.P.I. ",

19001. J. BRAINE-

" MAURICE CRAIG, M.D.

[The above form the Council.]

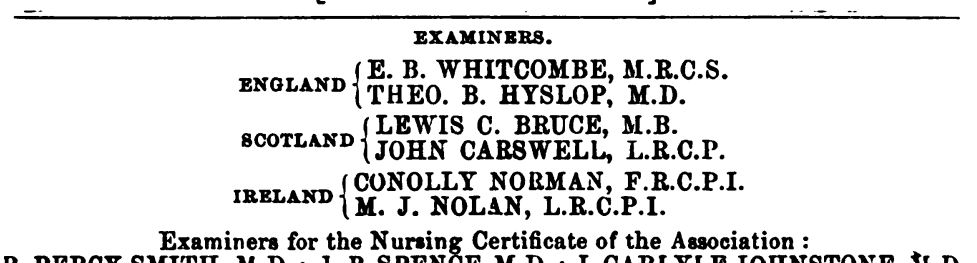

R. PERCY SMITH, M.D.; J. B. SPENCE, M.D. ; J. CARLYLE JOHNSTONE, JI.D.

PARLIAMETTARY COMMITPFE.

FLETCHER BEACH

H. BENHAM.

G. F. BLANDFORD.

DAVID BOWER.

D. M. CASSIDY.

T. S. CLOUSTON.

A. D. O'C. FINEGAN.

H. GARDINER HILL.

C. K. HITCHCOCK.

J. CARLYLE JOHNSTONE.

ROBERT JONES.

H. ROOKE LEY.

J. G. MoDOWALL.

C. MERCIER.
H. HAYES NEWINGTON

CONOLLY NORMAN.

EVAN POWELL.

H. KAYNER.

G. H. SAVAGE.

J. B. SPENCE.

A. H. S'TOCKER.

D. G. THOMPSON.

E. B. WHITCOMBE.

ERNEST W. WHITE.

J. WIGLESWORTH.

OSCAR WOODS.

D. YELLOWLEES. 
FDUCATIONAT COMMTTTEE.

T. s. CLOUSTON.

MAURICE CRAIG.

A. D. O'C. FINEGAN.

J. G. HAVELOCK.

T. B. HYSLOP.

J. CARLYLE JOHNSTONE.

ROBERT JONES.

W. S. KAY.

P. W. MACDONALD.

S. R. MACPHAIL.

T. W. MCDOWALL.

C. MERCIER (Secretary).

W. F. MICKLE.

G. W. MOULD.

H. HAYES NEWINGTON.

CONOLLY NORMAN.

H. RAYNER.
E. C. ROGERS.

J. RORIE.

G. H. SAVAGE.

T. CLAYE SHAW.

R. PERCY SMITH (Chairman).

J. B. SPENCE.

A. R. TURNBULLL

L. A. W EATHERLY.

E. B. WHITCOMBE.

ERNEST W. WHITE.

J. R. WHITWELL.

J. KENNEDY WILL.

OSCAR T. WOODS.

D. YELLOWLEES, and

THB PRESIDENT.

\section{LIST OF OHAIRMEN.}

1841. Dr. Blake, Nottingham.

1842. Dr. de Vitré, Lancaster.

1843. Dr. Conolly, Hanwell.

1844. Dr. 'Thurnam, York Retreat.

1847. Dr. Wintle, Warneford House, Oxford.

1851. Dr. Conolly, Hanwell.

1852. Dr. Wintle, Warneford House.

\section{LIST OF PRESIDENTS.}

1854. A. J. Sutherland, M.D., St. Luke's Hospital, London.

1855. J. Thurnam, M.D., Wilts County Asylum.

1856. J. Hitchman, M.D., Derby County Asylum.

1857. Forbes Winslow, M.D., Sussex House, Hammersmith.

1858. John Conolly, M.D., County Asylum, Hanwell.

1859. Sir Charles Hastings, D.C.L.

1860. J. C. Bucknill, M.D., Devon County Asylum.

1861. Joseph Lalor, M.D., Richmond Asylum, Dublin.

1862. John Kirkman, M.D., Sutfolk County Asylum.

1863. David Skae, M.D., Royal Edinburgh Asylum.

1864. Henry Munro, M.D., Brook House, Clapton.

1865. Wm. Wood, M.D., Kensington House.

1866. W. A. F. Browne, M.D., Commissioner in Lunacy for Scotland.

1867. C. A. Lockhart Robertson, M.D., Hnywards Heath Asylum.

1868. W. H. O. Sankey, M.D., Sandy well Park, Cheltenham.

1869. T. Laycock, M.D., Edinburgh.

1870. Robert Boyd, M.D., County Asylum, Wells.

1871. Henry Mudsley, M.D., The Lawn, Hanwell.

1872. Sir James Coxe, M.D., Commissioner in Lunacy for Scotland.

1873. Harrington Tuke, M.D., Manor House, Chiswick.

1874. T. L. Rogers, M.D., County Asylum, Rainhill.

1875. J. F. Duncan, M.D., Dublin.

1876. W. H. Parsey, M.D., Warwick County Asylum.

1877. G. Fielding Blandford, M.D., London.

1878. Sir J. Crichton-Browne, M.D., Lord Chancellor's Visitor.

1879. J. A. Lush, M.D., Fisherton House, Salisbury.

1880. G. W. Mould, M.R.C.S., Royal Asylum, Cheadle.

1881. D. Hack 'Tuke, M.D., London.

1882. Sir W. T. Gairdner, M.D., Glasgow. 
1883. W. Orange, M.D., State Criminal Lunatic Asylum, Broadmoor.

1884. Henry Rayner, M.D., County Asylum, Hanwell.

1885. J. A. Eames, M.D., District Asylum, Cork.

1886. Geo. H. Savage, M.D., Bethlem Royal Hospital.

1887. Fred. Needham, M.D., Barnwooil House, Gloucester.

1888. T. S. Clouston, M.D., Royal Edinburgh Asylum.

1889. H. Hayes Newington, M.R.C.P., Ticehurst, Sussex.

1890. David Yellowlees, M.D., Gartnavel Asylum, Glasgow.

1891. E. B. Whitcombe, M.R.C.S., City Asylum, Birmingham.

1892. Robert Baker, M.D., The Retreat, York.

1893. J. Murray Lindsay, M.D., County Asylum, Derby.

1894. Conolly Norman, F.R.C.P.I., Richmond Asylum, Dublin.

1895. David Nicolson, M.D., C.B., New Law Courts, Strand, W.C.

1896. William Julius Mickle, M.D., Grove Hall Asylum, Bow.

1897. Thomas W. McDowall, M.D., Morpeth, Northumberland.

1898. A. R. Urquhart, M.D., James Murray's Royal Asylum, Perth.

1899. J. B. Spence, M.D., Burntwood Asylum, nr. Lichfield, Staffordshire.

1900. Fletcher Beach, M.B., 79, Wimpole Street, W.

1901. Oscar T. Woods, M.D., District Asylum, Cork, Ireland.

\section{HONORARY MEMBERS.}

1896. Allbutt, T. Clifford, M.D., F.R.C.P., Regius Professor of Physic, Univ. Camb., St. Radegund's, Cambridge.

1881. Benedikt, Prof. M., Franciskaner Platz 5, Vienna.

1900. Blumer, G. Alder, M.D., L.R.C.P.Edin., Butler Hospital, Providence, U.S.A. (Ord. Mem., 1890.)

1900. Bresler, Johannes, M.D., Kraschnitz, Schlesien, Germany. (Corr. Mem., 1896.)

1881. Brosius, Dr., Bendorf-Sayn, near Coblenz, Germany.

1876. Browne, Sir J. Crichton-, M.D.Edin., F.R.S., Lord Chancellor's Visitor, New Law Courte, Strand, W.C. (Presidzst, 1878.)

1902. Brush, Edward N., M.D., Sheppard and Enoch Pratt Hospital, Towson, Maryland, U.S.A.

1887. Chapin, John B., M.D., Peunsylvania Hospital for the Insane, Philadelphia, U.S.A.

1902. Coupland, Sidney, M.D., F.R.C.P.Lond., Commissioner in Lunacy, 16, Queen Anne Street, Cavendish Square, London, W.

1872. Courtenay, E. Maziere, A.B., M.B., C.M.T.C.D., M.D., Inspector of 1872. \{ Lunatics in Ireland, Lunacy Office, Dublin Castle. (Secretary for Ireland, 1876-87.)

1879. Echeverria, M. G., M.D.

1865. Falret, Jules, M.D., 114, Rue de Bac, Paris.

1892. Féré, Dr. Charles, 37, Boulevard St. Michel, Paris.

1895. Ferrier, David, M.D., 34, Cavendish Square, London.

1872. Fraser, John, M.B., C.M., F.R.C.P.E., Commissioner in Lunacy, 19, Strathearn Road, Edinburgh.

1868. Gairdner, Sir William T., K.C.B., M.D.Edin., F.R.S., formerly Professor 1888. $\}$ of Medicine in the University of Glasgow, Physician to H.M. the King in Scotland, 32, George Square, Edinburgh. (Presidenx, 1882.)

1898. Hine, George T., F.R.I.B.A., 35, Parliament Street, London, S.W.

1881. Hughes, C. H., M.D., St. Louis, Missouri, United States. 
1866. Laehr, H., M.D., Schweizer Hof, bei Berlin, Editor of the Zeitschrift für Prychiatrie.

1887. Lentz, Dr., Asile d'Aliénés, Touruai, Belgique.

1898. MacDonald, A. E., M.D., Manhattan Asylum, New York, U.S.A.

1898. Magnan, V., M.D., Asile de Ste. Anne, Paris.

1871. Manning, Frederick Norton, M.D.St. And., M.R.C.S. Eng., Inspector of 1884. Asylums, 147, Macquarie Street North, Sydney, New South Wales. 1866. Mitchell, Sir Arthur, M.D.Aberd., LL.D., K.C.B., late Commissioner in

1871. Lunacy for Scotland; 34, Drummond Place, Edinburgh.

1897. Morel, M. Jules, M.D., States Lunatic Asylum, Mons, Belgium.

1880. Motet, M., 161, Rue de Charonne, Paris.

1889. Needham, Frederick, M.D.St. And., M.R.C.P.Edin., M.R.C.S.Eng., Commissioner in Lunacy, 19, Campden Hill Square, Kensington, W. (President, 1887.)

1891. O'Farrell, Sir G. P., M.D., M.Ch.Unir. Dubl., Inspector of Lunatics in Irelaud, 19, Fitzwilliam Sqnare, Dublin.

1881. Peeters, M., M.D., Gheel, Belgium.

1873. Pitman, Sir Henry A., M.D.Cantab., F.R.C.P.Lond., Registrar of the Royal College of Physicians, Enfield, Middlesex.

1900. Ritti, Ant., Maison Nationale de Charenton, St. Maurice, Paris. (Corr. Mom., 1890.)

1886. Roussel, M. Théophile, M.D., Sénateur, Paris.

1887. Schüle, Heinrich, M.D., Illenau, Baden, Germany.

1880. Sibbald, Sir John, M.D.Edin., F.R.C.P.Edin., M.R.C.S.Eng., Commissioner in Lunacy for Scotland ; 18, Great King Street, Edinburgh.
(Editor of Journal, 1871-2.)!

1888. Stearns, H. P., M.D., The Retreat, Hartford, Conn., U.S.A.

1881. Tamburini, A., M.D., Reggio-Emilia, Italy.

1901. Toulouse, Dr. Edonard, Editor of the Revue de Psychiatrie, Asile de Villejuif, Seine, France.

\section{CORRESPONDING MEMBERS.}

1896. Bianchi, Prof. Leonardo, Manicomio Provinciale di Napoli.

1897. Buschan, Dr. G., Stettin, Germany.

1896. Cowan, F. M., M.D., 107, Perponcher Straat, The Hague, Holland.

1902. Estense, Benedetto Giovanni Selvatico, M.D., 116, Piazza Porta Pia, Rome.

1880. Kornfeld, Dr. Hermann, Gleiwitz, Silesia, Germany.

1889. Kowalowsky, Professor Paul, Kharkoff, Russia.

1895. Lindell, Emil Wilhelm, M.D., Sweden.

1901. Manheimer-Gommès, Dr., 32, Rue de l'Arcade, Paris.

1897. Näcke, Dr. P., Hubertusberg Asylum, Leipzig.

1886. Parant, M. Victor, M.D., Toulouse.

1890. Régis, Dr. E., 54, Rue Huguerie, Bordeaux.

1893. Semelaigne, Dr. Réné, Secrétaire des Séances de la Société Módico. Psychologique de Paris, 16, Avenue de Madrid, Neuilly, Seine, France. 


\section{MEMBERS OF THE ASSOCIATION.}

Alphabetical List of Members of the Association, with the year in which they joined. The Asterisk means Members who joined between 1841 and 1855.

1900. Abbott, Arthur J., M.D., B.Ch., B.A.O., T.C.Dublin, Hants County Asylum, Fareham.

1900. Abbott, Henry Kingswell, M.B., B.Ch., M.D.Inblin, D.P.H.Ireland. Hants County Asylum, Fareham.

1891. Adair, Thomas Stewart, M.V., C.M.Edin., Assistant Medical Officer and Pathologist, Wadsley Asylum, near Sheffield.

1874. Adam, James, M.D.St. And., West Malling, Kent.

1868. Adams, Josiah O., M.D.Durh., F.R.C.S.Eng., Brooke House, Upper Clapton, London.

1880. Agar, S. H., L.R.C.P.I., Glendossil, Henley-in-Ardeu.

1886. Agar, S. Hollingsworth, juu., B.A.Cantab., M.R.C.S., Glendossil, Henleyin-Arden.

1901. Aherm, John M., L.R.C.P.\&S.I., Assistant Medical Officer, Warneford Asylum, Oxford.

1869. Aldridge, Chas., M.D.Aber., L.R.C.P., Plympton House, Plympton, Devon.

1899. Alexander, Hugh de Maine, M.D., The Hospital, Royal Asylum, Aberdeen.

1890. Alexander, Robert Reid, M.D.Aber., Medical Superintendent, Hanwell Lunatic Asylum.

1882. Alliott, A. J., M.D., Rosendal, Sevenoaks.

1899. Allmann, Dorah Elizabeth, M.B., B.Ch., B.A.O.R.U.I., Assistant Medical Officer, District Asylum, Armagh.

1901. Ambler, John Richardson, M.R.C.S., L.R.C.P.Lond., Senior Assistant Medical Officer, County Asylum, Chester.

1885. Amsden, G., M.B., Medical Supt., County Asylum, Brentwood, Essex.

1901. Anderson, James, M.B., C.M., Assistant Medical Officer, County Asylum, Winterton, Ferry hill, Durham.

1900. Anderson, John Charles, M.D.Durh., Darenth Asylum, Dartford, Kent.

1898. Anderson, John Sewell, M.R.C.S., L.R.C.P., Assistant Medical Officer, Hull City Asylum, Willerby, near Hull.

1901. Anderson, W. C., M.B., C.M., Fife and Kinross District Asylum, Cupar, Fife.

1894. Andriezen, W. Lloyd, M.D.Lond., 7, Apsley Terrace, Acton, W.

1894. Angus, Charles, M.B., C.M., Royal Infirmary, Aberdeen.

1898. Astbury, Thomas, M.R.C.S., L.R.C.P., Market Bosworth, near Nnneaton.

1892. Atherstone, Walter H., M.D., Surgeon-Superintendent, Port Alfred Asylum, South Africa.

1891. Aveline, Henry T. S., M.R.C.S., L.R.C.P., M.P.C., Medical Superintendent, County Asylum, Cotford, near Taunton, Somerset.

1894. Baily, Percy J., M.B.Edin., Senior Assistant Medical Officer, London County Asylum, Hanwell, W.

1878. Baker, H. Morton, M.B.Edin., Assistant Medical Officer, Leicester Borough Asylum, Humberstone, Leicester.

1888. Baker, John, M.D., Deputy Superintendent, State Asylum, Broadmoor, Berks.

1876. Baker, Robert, M.D.Edin., Visiting Physician, The Retreat, York, 41, The Mount, York. (Prasidrnt, 1892.)

1900. Barnes, Joseph Sandert, M.R.C.S.Eng., L.R.C.P.Lond., 3, Lyndhurst Square, Peckham, S.E.

1901. Barnett, Horatio, M.B., B.C.Cuntab., M.R.C.S., L.R.C.P.Lond., Medical Superintendeut, Stretton House, Cunrch Stretton, Salop.

1895. Barraclough, Herbert, M.B., The Asylum, Parirua, nr. Wellington, New Zealand. 
1878. Barton, James Edward, L.R.C.P.Edin., L.M., M.R.C.S., Medical Superintendent, Surrey County Lunatic Asylum, Brookwood, Woking.

1901. Barwell, Francis B., M.R.C.S.Eng., L.R.C.P.Lond., Assistant Medical Officer, Darenth Asylum, Dartford, Kent.

1901. Baskin, J. Longheed, L.R.C.P.\&S.Edin., L.F.P.S.Glas., Assistant Medical Officer, County Asylum, Exminster, Devon.

1902. Baugh, Leonard D. H., M.B., C.M., District Asylum, Larbert, Stirling, N.B.

1864. Bayley, J., M.R.C.S., Medical Superintendent, St. Andrew's Hospital, Northampton.

1893. Bayley, Joseph Herbert, M.B., C.M.Edin., Assistant Medical Officer, St. Andrew's Hospital, Northainpton.

1874. Beach, Fletcher, M.B., F.R.C.P.Lond., forinerly Medical Superintendent, Darenth Asylum, Dartford; Winchester House, Kingston Hill, Surrey, and 79, Wimpole Street, W. (General Secretary, 18891896. President, 1900-1901.)

1892. Beadles, Cecil F., M.R.C.S., L.R.C.P., Assistant Medical Officer, Colney Hatch Asylum.

1902. Beale-Browne, Thomas Richard, M.R.C.S.Eng., L.R.C.P.Lond., Berrywood, Northampton.

1896. Beamish, George, L.R.C.S.I., L.R.C.P.E., L.M., Medical Officer's House, H.M. Prison, Wandsworth, London, S.W.

1881. Benham, H. A., M.D., Medical Superintendent, City and County Asylum, Stapleton, near Bristol.

1899. Beresford, Edwyn H., M.R.C.S. \& M.R.C.P.Lond., Tooting Bec Asylum, Tooting, S.W.

1894. Bernard, Walter, F.R.C.P.I., M.R.C.S.Eng., 14, Queen Street, Londonderry.

1894. Blachford, James Vincent, M.B., B.S.Durham, Assistant Medical Officer, Bristol Asylum, Fishponds, near Bristol.

1899. Blackwood, Catherine Mabel, L.R.C.P.\&S., L.F.P.\&S.Glasg., Wadsley Asylum, near Sheffield.

1898. Blair, David, M.A., M.B., C.M., County Asylum, Lancaster.

1883. Blair, Robert, M.D., 30, Queen's Square, Strathbango, Glasgow.

1901. Blake, Thomas Frederick Hillyer, L.R.C.P.\&S.Edin., Wakefield Road, Ackworth Moor 'Top, near Pontefract, Yorks.

1902. Blakiston, Frederick C., M.R.C.S., L.R.C.P., Claremont Rise, Seaford, Sussex.

1857. Blandford, George Fielding, M.D.Oxon., F.R.C.P.Lond., 48, Wimpole Street, W. (Presidrat, 1877.)

1897. Blandford, Joseph John Guthrie, B.A., D.P.H.Camb., M.R.C.S.Eng., L.R.C.P.Lond., Assistant Medicul Officer, County Asylum, Whittingham, Preston, Lancs.

1888. Blaxland, Herbert, M.R.C.S., Medical Superintendent, Callan Park Asslum, New South Wales.

1897. Bois, Charles A., L.R.C.S., L.R.C.P.Edin., Waverley Lodge, St. Saviours Road, Jersey.

1900. Bolton, Joseph Shaw, M.D., B.S., B.Sc.Lond., Claybury Hall, Woodford Bridge, Essex.

1892. Bond, Charles Hubert, D.Sc., M.D., Ch.M.Edin., Senior Assistant Medical Officer, London County Asylum, The Heath, Bexley, Kent.

1877. Bower, David, M.D.Aber., Springfield House, Bedford.

1877. Bowes, John Ireland, M.R.C.S.Eng., L.S.A., Medical Superintendent, County Asylum, Devizes, Wilts.

1893. Bowes, William Henry, M.D.Lond., Assistant Medical Officer, Plymouth Borough Asylum, Ivybridge, Devon.

1900. Bowles, Alfred, M.R.C.S., L.R.C.P., 10, South Cliff, Eastbourne.

1896. Boycott, A. N., M.D.Lond., M.R.C.S.Eng., L.R.C.P.Lond., Medical Superintendent, Herts County Asylum, Hill End, St. Albsns, Herts.

1898. Boyle, A. Helen A., M.D., 3, Palmeira Terrace, Hove, Brighton. 
1883. Boys, A. H., L.R.C.P.Edin., Chequer Lawn, St. Albans.

1891. Braine-Hartnell, George, L.R.C.P.Lond., M.R.C.S.Eng., Medical Superintendent, County and City Asylum, Powick, Worcester.

1893. Bramwell, John Milne, M.B., C.M.Edin., 15, Stratford Place, Oxford Street, W.

1881. Brayn, R., L.R.C.P.Lond., Medical Superintendent, Broadmoor Asylum, Crow thorne, Berks.

1895. Briscoe, John Frederick, M.R.C.S.Eng., Resident Medical Superintendent, Westbrooke House Asylum, Alton, Hants.

1892. Bristowe, Hubert Carpenter, M.D.Lond., Wrington, R.S.O., Somerset.

1893. Bruce, Lewis C., M.B.Edin., Druid Park, Murthly, N.B.

* Brushfield, Thomas N., M.D.St. And., Budleigh Salterton, Devon.

1896. Babb, Willian, M.R.C.S., L.R.C.P.Lond., Second Assistant Medical Officer, Worcester County Asylum, Powick, near Worcester.

1892. Bullen, Frederick St. John, M.R.C.S.Eng., 12, Pembroke Road, Clifton, Bristol.

1869. Burman, Wilkie J., M.D.Edin., Ramsbury, Hungerford, Berks.

1891. Caldecott, Charles, M.B., B.S.Lond., M.R.C.S., Medical Superintendent, Earlswood Asylum, Redhill, Surrey.

1889. Callcott, J. T., M.D., Medical Superintendent, Borough Asylum, Newcastle-on-Tyne.

1874. Cameron, John, M.D.Edin., Medical Superintendent, Argyll and Bute Asylum, Lochgilphead.

1902. Campariole, Paul Clem, M.B., C.M.Ed., Junior Assistant Medical Officer, County Asylum, Melton, Suffolk.

1894. Campbell, Alfred Walter, M.D.Edin., Pathologist, County Asylum, Rainhill, near Prescot, Lancashire.

1880. Campbell, P. E., M.B., C.M., Senior Assistant Medical Officer, District Asylum, Caterham.

1897. Campbell, Robert Brown, M.B., C.M.Edin., Assistant Medical Officer, Crichton Royal Institution, Dumfries, N.B.

1897. Cappe, Herbert Nelson, M.R.C.S.Eng., L.R.C.P.Lond., Assistant Medical Officer, Surrey County Asylum, Brookwood.

1891. Carswell, John, L.R.C.P.Edin., L.F.P.S.Glasg., Certifying Medical Officer, Barony Parish, 5, Royal Crescent, Glasgow.

1896. Cashman, James, M.B., B.Ch., B.A.O.Rojal Univ. Irel., Assistant Medical Officer, Cork District Asylum.

1902. Cassells, Alexsnder Henderson, M.B., Ch.B.Glasg., Senior Assistant Medical Officer, District Asylum, Sunnyside, Montrose.

1874. Cassidy, D. M., M.D., C.M.McGill Coll., Montreal, D.Sc̀. (Public Health) Edin., F.R.C.S.Edin., Medical Superintendent, County Asylum, Lancaster.

1888. Chambers, James, M.D., M.P.C., The Priory, Roehampton.

1865. Chapman, Thomas Algernon, M.D.Glas., L.R.C.S.Edin., Betula, Reigate.

1880. Christie, J. W. Stirling, M.D., Medical Superintendent, County Asylum, Stafford.

1878. Clapham, Wm. Crochley S., M.D., M.R.C.P., The Grbles, Mayfield, Sussex.

1879. Clarke, Henry, L.R.C.P.Lond., H.M. Prison, Wakefield.

1901. Cleland, William Lennox, M.B., B.Ch.Edin., Park Side, South Australia.

1862. Clouston, T. S., M.D.Edin., F.R.C.P.Edin., F.R.S.E., Physician Superintendent, Royal Asylum, Morningside, Edinburgh. (Editor of Journal, 1873-1881.) (PREsIDENT, 1888.) 
1879. Cobbold, C. S. W., M.D., The Elms, Batheaston, Bath.

1900. Coffey, Patrick, L.R.C.P.\&S.I., District Asylum Limerick, Ireland.

1892. Cole, Robert Henry, M.D.Lond., M.R.C.P.Lond., 48, Upper Berkeley Street, $\mathbf{W}$.

1900. Cole, Sydney John, M.A., M.D., B.Ch.Oxon., Wilts County Asylum, Devizes.

1896. Coles, Richard Ambrose, Barham, near Canterbury.

1902. Collie, Robert John, M.D., Assistant Medical Officer School Board for London, for Mentally Deficient Children, 25, Porchester Terrace, Hyde Park, $W$.

1888. Cones, John A., M.R.C.S., Burgess Hill, Sussex.

1895. Conry, John, M.D.Aber., Fort Benufort Asylum, South Africr.

1900. Cook, John Benson, L.R.C.P.\&S.Ed., Medical Officer H.M. Prison, Borstal, Rochester.

1878. Cooke, Edward Marriott, M.D., M.R.C.S.Eng., Commissioner in Lunacy, 69, Onslow Square, S.W.

1899. Cooke, J. A., Medical Officer and Co-Licensee, Tue Brook Villa, near Liverpool.

1902. Cooke, William Arthur, L.R.C.P.\&S.I., Assistant Medical Officer, St. Putrick's Hospital, Dublin.

1901. Cooper, K. D., M.R.C.S.Eng., Assistant Medical Officer, The Lawn, Lincoln.

1891. Corner, Harry, M.B.Lond., M.R.C.S., L.R.C.P., M.P.C., Brooke House, Southgate, $\mathbf{N}$.

1897. Cotton, William, M.A., M.D.Edin., D.P.H.Cantab., 231, Gloucester Road, Bishopston, Bristol.

1893. Cowen, Thomus Phillips, M.B., B.S.Lond., Assistant Medical Officer, County Asylum, Lancaster.

1899. Cowper, Alfred, M.A., M.B., C.M.Edin., Valkenburg Asylum, Mowbray, Cape Town.

1884. Cox, L. F., M.R.C.S., Medical Superintendent, County Asylum, Denbigh.

1878. Craddock, F. H., B.A.Oxon., M.R.C.S.Eng., L.S.A., Medical Superintendent, County Asylum, Gloucester.

1892. Craddock, Samuel, M.R.C.S.Eng., Summerdale, Bath.

1893. Craig, Maurice, M.A., M.B., B.C.Cantab., M.R.C.P.Lond., Assistant Medical Officer, Bethlem Royal Hospital, Sonthwark.

1897. Cribb, Harry Gifford, M.R.C.S.Eng., L.R.C.P.Lond., Assistant Medical Officer, London County Asylum, Canehill, Surrey.

1898. Crookshank, F. G., M.D.Lond., M.R.C.S., L.R.C.P., 27, The Terrace, Barnes, S.W.

1894. Cullinan, Henry M., L.R.C.P.I., L.R.C.S.I., Second Assistant Medical Officer, Richmond District Asylum, Dublin.

1902. Curran, Michael, M.A., M.B., B.Ch., R.U.I., Assistant Medical Officer, St. Patrick's Hospital, Dublin.

1869. Daniel, W. C., M.D.Heidelb., M.R.C.S.Eng., Epsom, Surrey.

1899. Daunt, Elliot, M.R.C.S., L.R.C.P., D.P.H., Rosendal, Sevenoaks, Kent.

1896. Davidson, Andrew, M.D., C.M.Aber., Straits Settlements.

1874. Davies, Francis P., M.D.Edin., M.R.C.S.Eng., Kent County Asylum, Barming Heath. near Maidstone.

1891. Davis, Arthur N., L.R.C.P., L.R.C.S.Edin., Medical Superintendent, County Asylum, Exminster, Devon.

1894. Dawson, William R., M.D., B.Ch.Dubl., F.R.C.P.I., Medical Superintendent, Furnhain House Asylum, Finglas, Dublin. 
1869. Deas, Peter Maury, M.B. and M.S.Lond., Medical Superintendent, Wonford House, Exeter.

1900. Despard, Rosina C., M.D.Lond., Holloway Sanatorium, Virginia Water, Surrey.

1901. De Steiger, Adèle, M.B.Lond., Connty Asylum, Brentwood, Essex.

1876. Dickson, F. K., F.R.C.P.Edin., Wye House Lunatic Asylum, Buxton, Derbyshire.

1902. Dixon, Harry Livesuy, M.A., M.B., B.C., D.P.H.Cantab., Senior Assistant Medical Officer, County Asylum, Mickleover, Derby.

1879. Dodds, William J., M.D., D.Sc.Edin., Valkenburg, Mowbray, near Cape Town, South Africa.

1886. Donaldson, Robert Lockhart, B.A., M.D., B.Ch.Univ, of Dubl., M.P.C., Senior Medical Officer, District Asylum, Monaghan.

1889. Donaldson, William Ireland, B.A., M.D., B.Ch.Univ. of Dubl., Medical Superintendent, Horton Manor Asylum, Epsom, Surrey.

1892. Donelan, John O’Conor, L.R.C.P.I., L.R.C.S.I., M.P.C., First Assistant Medical Officer, Portrane Asylum, Donabate, co. Dnblin.

1899. Donelan, Thomas O'Conor, L.R.C.P. \& L.R.C.S.Ireland, Menston Asylum, near Leeds.

1891. Douglas, Archibald Robertson, L.R.C.S., L.R.C.P.Edin., Royal Albert Asylum, Lancaster.

1890. Douglas, William, M.D.Queen's Univ. Irel., M.R.C.S.Eng., Brandfold, Goudhurst.

1897. Dove, Emily Louisa, M.B.Lond., Cowbitt Vicarage, nr. Spalding.

1884. Drapes, Thomas, M.B., Medical Superintendent, District Asylum, Enniscorthy, Ireland.

1902. Dudgeon, Herbert Wm., M.D.Durh., M.R.C.S.Eng., L.R.C.P.Lond., Medical Officer to the Egyptian Asylum, Abassieh, Cairo, Egypt.

1899. Dudley, Francis, L.R.C.P.\&S.I., Senior Assistant Medical Officer, County Asylum, Bodmin, Cornwall.

1899. Eades, Albert J., County Asylum, Winwick, Warrington, Lancs.

1874. Eager, Reginald, M.D.Lond., M.R.C.S.Eng., Northwoods, near Bristol.

1873. Eager, Wilson, L.R.C.P.Lond., M.R.C.S.Eng., Northwoods, Winterbourne, Bristol.

1881. Earle, Leslie, M.D.Edin., 108, Gloucester Terrace, Hyde Park, W.

1891. Earls, James Henry, M.D., M.Ch., \&c., 71, Brighton Square, Dublin.

1895. Easterbrook, Charles C., M.A., M.D., M.K.C.P.Ed., Medical Superintendent, Ayr District Asylum, Glengail, Ayr, N.B.

1895. Edgerly, Samuel, M.B., C.M.Edin., Assistant Medical Officer, West Riding Asylum, Menston, nr. Leeds.

1900. Edridge-Green, F. W., M.D., F.R.C.S., Hendon Grove, Hendon, N.W.

1902. Edwards, Charles, M.R.C.S., L.R.C.P., Assistant Medical Officer, City of London Asylum, nr. Dartford, Kent.

1897. Edwards, Francis Henry, M.D.Brux., M.R.C.P.Lond., Medical Superintendent, Camberwell House, S.E.

1901. Elgee, Samuel Charles, L.R.C.P., L.R.C.S.Ire., Assistant Medical Officer, Horton Manor Asylum, Epsom, Surrey.

1889. Elkins, Frank Ashley, M.D., Medical Superintendent, Metropolitan Asylum, Leavesden.

1898. Ellerton, H. B., M.R.C.S., L.R.C.P., County Asylum, Nottingham.

1873. Elliot, G. Stanley, M.R.C.P.Edin., F.R.C.S.Edin., 16, Killieser Avenue, Streatham Hill, S.W.

1900. Ellis, Henry Reginald, M.R.C.S., L.R.C.P.Lond., Shipley Hall, Shipley, Yorks. 
1890. Ellis, William Gilmore, M.D.Brux., Superintendent, Government Asylum, Singapore.

1899. Ellison, Fras. C., M.B., B.Ch., T.C.D., Assistant Medical Officer, District Asylum, Castlebar.

1901. Elsworth,T. G., M.B., C.M.Edin., Senior Assistant Medical Officer, County and City Asylum, Hereford.

1901. Erskine, Wm. J. A., M.D., C.M., Senior Assistant Medical Officer, City Asylum, Nottingham.

1895. Eurich, Frederick William, M.D., C.M.Edin., 7, Lindum Terrace, Bradford, Yorks.

1894. Eustace, Henry Marcus, M.B., B.Ch., B.A.Univ. Dublin, Assistant Physician, Humpstead and Highfield Private Asylum, Glasnevin, Dublin.

1901. Evaus, James Wm., M.R.C.S., L.S.A., Lieut.-Col. Indian Medical Service (retired), Tattlebury House, Goudhurst, Kent.

1897. Everett, William, M.D., Assistant Medical Officer, County Asylum, Chartham Downs, Kent.

1891. Ewan, John Alfred, M.A., M.B., C.M.Edin., M.P.C., Greylees, Sleaford, Lincolnshire.

1884. Ewart, C. T., M.B., C.M.Aberd., Claybury Asylum, Woodford Bridge, Essex.

1894. Farquharson, William F., M.D.Edin., Medical Superintendent, Counties Asylum, Garlands, Carlisle.

1901. Fee, Wm. George, L.R.C.P. and L.R.C.S.Edin., Assistant Medical Officer, Brooke House, Upper Clapton, N.E.

1897. Fielding, James, M.D., Victoria Univ., Canada, M.R.C.S.Eng., L.R.C.P. Edin., Modical Superintendent, Bethel Hospital, Norwich.

1873. Finch, John E. M., M.D., Medical Superintendent, Borough Asylum,

1889. Finch, Richard T., B.A., M.B.Cantab., Resident Medical Officer, Fisherton House Asylum, Sulisbury.

1867. Finch, W. Corbin, M.R.C.S.Eng., Fisherton House, Salisbury.

1901. Findlay, John, M.B., Ch.B.Aber., Assiatant Medical Officer, County Asylum, Dorchester, Dorset.

1882. Finegan, A. D. O'Connell, L.R.C.P.I., Medical Superintendent, District Asylum, Mullingar. (Hon. Secretary for Ireland.)

1889. Finlay, David, M.D.Glasg., County Asylum, Bridgend, Glamorgan.

1898. Finn, P. Tasffe, L.R.C.P., L.R.C.S.Ed., Oakhill, nr. Bath.

1894. Fitzgerald, Charles E., M.D., F.R.C.S.I., Surgeon-Oculist to the Queen in Ireland, 27, Upper Merrion Street, Dublin.

1888. Fitzgerald, G. C., M.B., B.C.Cantab., M.P.C., Medical Superintendent, Kent County Asylum, Chartham, nr. Canterbury.

1899. Fitzgerald, James J., M.B., B.Ch., B.A.O.R.U.I., Assistant Medical Officer, District Asylum, Carlow.

1901. Fitzgerald, John J., M.D.Brux., L.R.C.P.\&S.Edin., Assistant Medical Officer, District Asylum, Cork.

1900. Fleck, David, M.B., Ch.B., B.A.O.Ireland, The Asylum, Caterham, Surrey.

1899. Flemming, A. L., M.R.C.S.Eng., L.R.C.P.Lond., City and County Asylum, Fishponds, Bristol.

1872. Fletcher, Robert Vicars, Esq., F.R.C.S.I., L.R.C.P.I., L.R.C.P. Edin., Medical Supt., District Asylum, Bullinasloe, Ireland.

1894. Fleury, Eleonora Lilian, M.D., B.Ch., R.U.I., Assistant Medical Officer, Richmond Asylum, Dublin.

1902. Forde, Michael J., M.D., M.Ch., R.U.I., Assistant Medical Officer, Richmond Asylum, Donabate, Dublin.

1902. Forshaw, W m. H., M.R.C.S., L.R.C.P.Lond., 29, Tredegar Square, Bow, E.

1902. Forster, Hermann Julius, L:R.C.P.I., L.S.A., Assistant Medical Officer, East Sussex Asylum, Hayward's Heath. 
1899. Forsyth, Charles E. P., M.B., Ch.B., Eastern Hospital, The Grove, Homerton, N.E.

1902. Forsyth, John Glen, M.B., C.M.Ed., c/o Dr. James Forsyth, Eyemouth, Berwickshire.

1861. Fox, Charles H., M.D.St. And., M.R.C.S.Eng., 35, Heriot Row, Edinburgh.

1896. Frunce, Eric, M.B., B.S.Durh., Assistant Medical Officer, Claybury Asylum, Woodford Bridge, Essex.

1881. Fraser, Donald, M.D., 3, Orr Square, Paisley.

1901. French, Louis Alexander, M.R.C.S., L.R.C.P., 104, Church Road, Silverdale, Staffs.

1902. Fuller, Lawrence Otway, M.R.C.S.Eng., L.R.C.P.Lond., Assistant Medical Officer, Darenth Asylum, Dartford, Kent.

1893. Garth, H. C., M.B., C.M.Edin., 4, Harrington Street, Calcutta, India.

1890. Gaudin, Francis Neel, M.R.C.S., L.S.A., M.P.C., Medical Superintendent, The Grove, Jersey.

1885. Gayton, Francis C., M.D., Brookwood Asylum, Woking, Surrey.

1896. Geddes, John W., M.B., C.M.Edin., Assistant Medical Officer, Durham County Asylum, Winterton, Ferryhill, Durham.

1892. Gemmel, James Francis, M.B.Glasg., Assistant Medical Officer, County Asylum, Whittingham, Preston.

1889. Gibbon, William, L.R.C.P.I., L.F.P.S.Glasg., Senior Assistant Medical Officer, Joint Counties Asylum, Carmarthen.

1899. Gilfillan, Samuel James, M.A., M.B.Edin., London County Asylum, Canehill, Purley, Surrey.

1898. Gill, Frank A., M.D., C.M.Aber., Deputy Medical Officer, H.M. Prison, Liverpool.

1889. Gill, Stanley, B.A., M.D., M.R.C.P.Lond., Shaftesbury House, Formby, Lancashire.

1897. Gilmour, John Rutherford, M.B., C.M.Edin., West Riding Asylum, Scalebor Park, Burley-in-Wharfedale, Yorks.

1901. Glasgow, John George, M.R.C.S.Eng., L.R.C.P.Lond., Assistant Medical Officer, Borough Asylum, Portsmouth.

1878. Glendinning, James, M.D.Glasg., L.R.C.S.Edin., L.M., Medical Superintendent, Joint Counties Asylum, Abergavenny.

1898. Goldie-Scot, Thomas, M.B., C.M.Edin., M.R.C.S., L.R.C.P., Junior Assistunt Physician, Royal Asylum, Gartnavel, Glasgow.

1899. Goldschmidt, Oscar Bernard, M.B., Ch.B.Vict., Durham House, Withington, Manchester.

1897. Good, Thomas Saxty, M.R.C.S.Eng., L.R.C.P.Lond., Assistant Medical Officer, County Asylum, Littlemore, Oxford.

1889. Goodall, Edwin, M.D., M.S.Lond., M.P.C., Medical Superintendent, Joint Counties Asylum, Carwarthen.

1899. Goodliffe, John Henry, Finbar House, 802, High Road, Lower Totten. ham, $N$.

1899. Gordon, J. Leslie, M.B., Ch.B., County Asylum, Devizes, Wilts.

* Gordon, W. S., M.B., District Asylum, Mullingar.

1901. Gostwyck, C. H. G., M.B., Ch.B., Medical Officer, Kent Lunatic Asylum, Charthain Downs, nr. Cunterbury.

1899. Graham, R. A. L., B.A., M.B., B.Ch., R.U.I., Assistant Medical Officer, District Asylum, Belfast.

1894. Graham, Samuel, L.R.C.P.Lond., Assistant Medical Officer, District Asylum, Antrim.

1888. Grahum, T., M.D.Glasg., 3, Garthland Place, Paisley.

1887. Graham, W., M.D., R.U.I., Medical Superintendent, District Lunatic Asylum, Belfast. 
1890. Gramshaw, Farbrace Sidney, M.D., L.R.C.P.I., L.R.C.S.Edin., L.M., L.A.H.Dubl., The Villa, Stillington, Yorkshire.

1897. Grant-Wilson, Charles Westbrook, L.R.C.P.Lond., M.R.C.S.Eng., St. Winnows, Bromley, Kent.

1902. Green, Philip A. M., M.R.C.S., L.R.C.P., Assistant Medical Officer, Claybury Asylum, Woodford Bridge, Essex.

1902. Greene, George Waters, B.A., M.B.Cantab., M.R.C.S., L.R.C.P., Assistant Medical Officer, Claybury Asylum, Woodford Bridge, Essex.

1896. Greene, Thomas Adam, Assistant Medical Officer, District Asylum, Ennis, Ireland.

1886. Greenlees, T. Duncan, M.B., Medical Superintendent to the Grahamstown Asylum, Cape of Good Hope.

1894. Griffin, Edward W., M.D., M.Ch., R.U.I., Assistant Medical Officer, The Asylum, Killarney.

1896. Griffiths, George Batlio G., M.R.C.S., L.R.C.P.Lond., Assistant Surgeon, H.M. Convict Prison, Parkhurst, Isle of Wight.

1901. Grills, Galbraith Hamilton, M.B., B.Ch., Assistant Medical Officer, County Asylum, Chester.

1900. Grove, Ernest George, M.R.C.S., L.R.C.P., York Lunatic Hospital, Bootham, York.

1894. Gwynn, Charles Henry, M.D.Edin., co-Licensee, St. Mary's House, Whitchurch, Salop.

1879. Gwynn, S. T., M.D., St. Mary's House, Whitchurch, Salop.

1894. Halstead, Harold Cecil, M.D.Durh., Assistant Men̈ical Officer, Peckham House, Peckham.

1902. Hanbury, Saville Waldron, M.R.C.S.Eng., L.R.C.P.Lond., Assistant Medical Officer, Loudon County Asylum, Banstead, Surrey.

1896. Hanbury, William Reader, M.R.C.S., L.R.C.P., Senior Assistant Medical Officer, West Ham Borough Asylum, Goodmayes, Ilford.

1901. Hannay, Mary Baird, M.B., C.M., Gartloch Asylum, Gartcosh, Glasgow, N.B.

1901. Harding, William, M.D., M.R.C.P.Lond., Medical Superintendent, Northampton County Asylum, Berry Wood, Northampton.

1899. Harmer, W.A., L.S.A., Resident Superintendent and Licensee, Redlands Private Asylum, 'Tonbridge, Kent.

1895. Harper, Thomas Edward, L.R.C.P.Lond., M.R.C.S.Eng., Assistant Medical Officer, St. Ann's Heath, Virginia Water.

1897. Harris, William, M.D.St. And., F.R.C.S.Edin., M.R.C.P.Edin., Medical Superintendent, City Asylum, Hellesdon, Norwich.

1898. Harris-Liston, L., M.D., M.R.C.S., L.R.C.P.Lond., L.S.A., City Asylum, Digbys, Exeter.

1886. Harvey, Crosbie Bagenal, L.A.H., Assistant Medical Officer, District Asylum, Clonmel.

1892. Haslett, William John, M.R.C.S., L.R.C.P., Resident Medical Superintendent, Halliford House, Sunbury-on-Thames.

1891. Havelock, John G., M.B., C.M.Edin., Physician Superintendent, Montrose

Royal Asylum.
1890. Hay, Frank, M.B., C.M., Physician Superintendent, Ashburn Hull Asylum, Dunedin, New Zealand.

1900. Haynes, Horace E., M.R.C.S., L.S.A., Bishopstow House, Bedford.

1895. Hearder, Frederic P., M.D., C.M., Assistant Medical Officer, West Riding Asylum, Wakefield.

1885. Henley, E. W., L.R.C.P., County Asylum, Barnwood, Gloucester.

1899. Herbert, W. W., M.D., C.M.Edin., North Wales Counties Asylum, Deubigh, North Wales.

1877. Hetherington, Charles, M.B., Medical Superintendent, District Asylum, Londonderry, Ireland.

1877. Hewson, R. W., L.R.C.P.Edin., Medical Superintendent, Cotton Hill, Stafford. 
1902. Higginson, John Wigmore, M.R.C.S., L.R.C.P., Resident Medical Otticer, Hayes Park Asylum, Hayes Park, Middlesex.

1882. Hill, Dr.H. Gardiner, Medical Superintendent, Middlesex County Asylum, Tooting.

1900. Hill, J. R., M.R.C.S., L.R.C.P., Fenstanton, Christchurch Road, Streatham Hill, S.W.

1902. Hingston, A. Alwyne, B.A.Cantab., M.B., C.M.Aberd., Assistaut Medical Officer, Cotford Asylum, Taunton.

1871. Hingston, J. Tregelles, M.R.C.S.Eng., Medical Superintendent, North Riding Asylum, Clifton, Yorks.

1881. Hitchcock, Charles Knight, M.D., Bootham Asylum, York.

1900. Holländer, Bernard, M.D., M.R.C.S.. L.R.C.P., 62, Queen Anne Street, London, $\mathbf{W}$.

1896. Horton, James Henry, M.R.C.S.Eng., L.R.C.P.Lond., Lieut. I.M.S., c/o Messrs. W. Wutson \& Co., 7, Waterloo Place, S.W.

1894. Hotehkis, R. D., M.D., C.M., M.P.C., Assistunt Physician, Royal Asylum, Glasgow.

1900. Hughes, Percy T., M.B., Ch.M.Edin., London County Asylum, Bexley, Kent.

1900. Hughes, George Osborne, M.D.Virginia, M.R.C.S., L.R.C.P., 16, Harvey Road, Hornsey, London, N.

1857. Humphry, J., M.R.C.S.Eng.: Medical Superintendent, County Asylum, Stoile, near A ylesbury, Bucks.

1897. Hunter, David, M.A., M.B., B.C.Cantab., West Ham Borough Asylum. Goodmayes, Ilford, Essex.

1882. Hyslop, James, D.S.O., M.D., c/o Dr. Cullen Brown, Overton Park, Alexandria, Dumbartonshire, N.B.

1888. Hyslop, Theo. B., M.D., C.M.Edin., M.R.C.P.E., M.P.C., Bethlem Roynl Hospital, S.E.

1871. Ireland, W. W., M.D.Edin., 1, Victoria Terrace, M usselburgh, N.B.

1866. Jackson, J. Hughlings, M.D.St. And., F.R.C.P.Lond., F.R.S., Physician to the Hospitul for Epilepsy and Paralysis, \&c., 3, Manchester Square, London, $W$.

1893. Johnston, Geruld Herbert, L.R.C.S. and L.R.C.P.Edin., Ticehurst House, Sussex.

1878. Johnstone, J. Carlyle, M.D., C.M., Medical Superintendent, Roxburgh District Asylum, Melrose.

1880. Jones, D. Johnson, M.D.Edin., Medical Superintendent, Banstead Asylum, Surrey.

1866. Jones, Evan, M.R.C.S.Eng., Ty-mawr, Aberdare, Glamorganshire.

1882. Jones, Robert, M.D.Lond., B.S., F.R.C.S., Medical Superintendent, London County Asylum, Claybury, Woodford, Essex. (Gen. Secretary from 1897.)

1897. Jones, Samuel Lloyd, M.R.C.S.Eng., L.R.C.P.Lond., Assistant Medical Officer, London County Asylum, Colney Hatch, $\mathbf{N}$.

1898. Jones, W. Ernest, M.R.C.S.Eng., L.R.C.P.Lond., Brecon and Radnor Asylum, Talgarth, R.S.O.

1897. Jones, William Edward, Assistant Medical Officer, Earlswood Asylum, Redhill, Surrey.

1879. Kay, Walter S., M.D., Medical Superintendent, South Yorkshire Asylum, Wadsley, near Sheffield.

1886. Keay, John, M.B., Medical Superintendent, District Asylum, Inverness.

1899. Keegan, Lawrence Edward, M.D., Medical Superintendent, Lunatic Asylum, St. John's, Newfoundland.

1902. Kelley-Patterson, Wm., M.D., M.Ch., R.U.I., Bally-Emond, Killowen, Dublin.

1898. Kemp, Norah, M.B., C.M.Glas., The Rotreat, York.

1899. Kennedy, Hugh T. J., L.R.C.P.\&S.I., L.M., Assistant Medical Officer, District Asylum, Enniscorthy. 
1902. Kennedy, Patrick Gabriel, L.R.C.P.\&S.Edin., L.F.P.S.Glasg., Assistant Medical Officer, London County Asylum, Bunstead, Surrey.

1897. Kerr, Hugh, M.A., M.D.Glasg., Assistant Medical Officer, Bucks County Asylum, Stone, Aylesbury, Bucks.

1902. Kerr, Neil Thomson, M.B., C.M.Ed., Medical Superintendent, Lanark District Asylum, Hartwood, Shotts, N.B.

1893. Kershaw, Herbert Warren, M.R.C.S.Eng., L.R.C.P.Lond., Dinsdale Park, near Darlington.

1897. Kidd, Harold Andrew, M.R.C.S.Eng., L.R.C.P.Lond., Medical Superintendent, West Sussex Asylum, Chichester.

1897. Kingdon, Wilfred Robert, M.B., B.S.Durh., 55, Haverstock Hill, London, N.W.

1902. King-Turner, A. C., M.B., C.M.Edin., The Retreat, Fairford, Gloucestershire.

1899. Kirwan, J. St. L., M.B., Ch.B., T.C.D., District Asylum, Ballinasloe, Ireland.

1898. Labey, Julius, M.R.C.S., The Myrtles, St. Saviour's, Jersey.

1900. Laing, Charles Frederick, M.B., C.M.Glasg., County Asylum, Wells, Somerset.

1900. Lambert, Ernest Charles, M.R.C.S.Eng., L.R.C.P.Lond., Banstead Asylum, Sutton, Surrey.

1902. Langdon-Down, Percival L., M.B., B.C.Cantab., Rudder Grange, Cedar Rond, Hampton Wick, Middlesex.

1896. Langdon-Down, Reginald L., M.B., B.C.Cantab., M.R.C.P.Lond., Normansfield, Hampton Wick.

1902. Laval, Evariste, M.B., C.M.Edin., Brislington House Asylum, near Bristol.

1898. Lavers, Norman, M.R.C.S., Medical Superintendent, The Asylum, Canterbury.

1899. Law, Charles D., L.R.C.P.\&S.Edin., L.F.P.G.S., c/o District Asylum, Inverness, N.B.

1892. Lawless, Dr. George Robert, A.M.O., District Asylum, Armagb.

1870. Lawrence, A., M.D., County Asylum, Chester.

1883. Layton, Henry A., L.R.C.P.Edin., Cornwall County Asylum, Bodmin.

1899. Leeper, R. R., F.R.C.S.I., Medical Superintendent, St. Patrick's Hospital, Dublin.

1883. Legge, R. J., M.D., Medical Superintendent, County Asylum, Derby.

1894. Lentagne, John, B.A., F.R.C.S.I., Medical Visitor of Lunatics to the Court of Chancery, 5, Upper Merrion Street, Dublin.

1899. Lewis, H. Wolseley, M.R.C.S.Eng., L.R.C.P.Lond., Banstead Asylum, Sutton, Surrey.

1879. Lewis, William Bevan, West Riding Asylum, Wakefield.

1863. Ley, H. Rooke, M.R.C.S.Eng., 2, Lowther Terrace, Lytham, Lancs.

1899. Ligertwood, Walter H., L.R.C.P., Wells Asylum, Somerset.

1900. Lindsay, David Lauder, L.R.C.P.\&S.Edin.

1859. Lindsay, James Murray, M.D.St.And., F.R.C.S. and F.R.C.P.Edin. 26, Combe Park, Bath. (Presidrat, 1893.)

1883. Lisle, S. Ernest de, L.R.C.P.I., Three Counties Asylum, Stotfold, Herts.

1899. Longworth, Stephen G., L.R.C.P. and S.I., County Asylum, Melton, Suffolk. 
1898. Lord, John R., M.B., C.M., Heath Asylum, Bexley, Kent.

1872. Lyle, Thomas, M.D.Glasg., 34, Jesmond Road, Newcastle-on-Tyne.

1899. Macartney, W. H. C., L.R.C.P.\&S.I., The Grange, East Finchley, London, $\mathbf{N}$.

1880. MacBryan, Henry C., Kingsdown House, Box, Wilts.

1901. Macdonald, J. H., M.B., Ch.B.Glitsg., Govan District Asylum, Hawkhead, Paisley, N.B.

1884. Macdonald, P. W., M.D., C.M., Medical Superintendent, County Asylum, near Dorchester, Dorset. (Hon. Sec. S.W. Division.)

1893. Macevoy, Henry John, M.D., B.Sc.Lond., M.P.C., 41, Buckley Road, Brondesbury, London, N.W.

1895. Macfarlane, Neil M., M.D.Aber., Medical Superintendent, Government Hospital, Thlotse Heights, Leribe, Basutoland, South Africa.

1883. Macfarlane, W. H., M.B. and Ch.B.Univ. of Melbourne, Medical Superintendent, Hospital for the Insane, New Norfolk, Tasmania.

1891. Mackenzie, Henry J., M.B., C.M.Edin., M.P.C.,Assistant Medical Officer, The Retreat, York.

1899. Mackeown, W. John, A.B., M.B., B.A , O.R.U.I., A.M.O., County Asylum, Fareham, Hants.

* Mackintosh, Donald, M.D.Durh. and Glasg., L.F.P.S.Glasg., 10, Lancaster Road, Belsize Park, N.W.

1873. Macleod, M. D., M.B., Medical Superintendent, East Riding Asylnm, Beverley, Yorks.

1901. Macleod, Neil, M.D., C.M.Edin., H.B.M. Consular Surgeon and Surgeou General, The Hospital, Shanghai, China.

1899. MacLulich, Peers, M.B., B.C., B.A.Dubl., c/o Dr. Goodall, Joint Counties Asylum, Carmarthen.

1898. Macnaughton, George W. F., M.D., Warwick Lodge, 436, Fulham Road, London, S.W.

1882. Macphail, Dr. S. Rutherford, Derby Borough Asylum, Rowditch, Derby.

1896. Macpherson, Dr. Charles, Deputy Commissioner in Lunacy, 51, Queen Street, Edinburgh.

1886. Macpherson, John, M.B., M.P.C., 8, Darnaway Street, Edinburgh.

1901. MacRae, Duncan M., M.B., C.M., County Asylum, Devizes, Wilts.

1902. Macrae, Kenneth Duncan Cameron, M.B., Ch.B.Edin., District Asylum, Inverness, N.B.

1895. Madge, Arthur E., M.R.C.S.Eng., L.R.C.P.Lond.

1896. Maguire, Charles Evan, M.B., C.M., District Medical Officer, Old Calabar, Southern Nigeria, W. C. Africa.

1896. Mallanah, S., M.B.Edin., Medical School, Hyderabad, Deccan, India.

1865. Manning, Harry, B.A.Lond., M.R.C.S., Laverstock House, Salisbury.

1900. Manning, Herbert C., M.R.C.S., L.R.C.P., Wye, Kent.

1896. Marr, Hamilton C., M.D.Glasg.Univ., Medical Superintendent, Woodilee Asylum, Lenzie.

1897. Marshall, John, M.B., C.M.Glasg., Assistant Medical Officer, County Asslum, Bridgend, Glamorgan.

1896. Martin, James Clurke, L.R.C.S.I., L.M., L.R.C.P., Assistant Medical Officer, District Asylum, Donegal.

1897. Mathieson, George, M.B., C.M.Glasg., Fir Vale, Sheffield.

1888. MeAlister, William, M.B., C.M., The Elms, Kilmarnock, N.B. 
1902. McCarthy, Owen F., L.R.C.P.\&S.I., District Lunatic Asylum, Cork, Ireland.

1900. McClintock, John, L.R.C.P. \& L.R.C.S.Edin., Resident Medical Superintendent, Grove House, Church Stretton, Salop.

1900. McConaghey, J. C., M.B., C.M.Edin., Purkside Asylum, Macclesfield, Cheshire.

1886. McCreery, James Vernon, L.R.C.S.I., Medical Superintendent, Hospital for Insane, Kew, Victoria.

1897. McCutchau, William Arthur, L.R.C.P.S.Edin., Assistant Medical Officer, Cambridge County Asylum, Fulbourn, Cambs.

1876. McDowall, John Greig, M.B.Edin., Medical Superintendent, West Riding Asylum, Menston, near Leeds.

1870. McDowall, T. W., M.D.Edin., L.R.C.S.E., Medical Superintendent, Northumberland County Asylum, Morpeth. (PResidrert, 1897.)

1902.' McGregor, John, M.B., Ch.B.Edin., Assistant Medical Officer, County Asylum, Fulbourn, Cambridge.

1899. McKelvey, Alezander Niel, L.\&M.P.C.P.\&S.I., New Zealand.

1882. McNuughton, John, M.D., Medical Superintendent, Criminal Lnnatic Asylum, Perth.

1901. McRae, G. Douglas, M.B., C.M.Edin., Assistant Physician, Royal Asylum, Morningside, Ediuburgh.

1894. McWilliam, Alexander, M.B., C.M.Aber., Medical Superintend ent, Heigham Hall, Norwich.

1890. Menzies, W. F., M.D., B.Sc.Edin., Medical Superintendent, Stafford County Asylum, Cheddleton, near Leek.

1891. Mercier, Charles A., M.B.Lond., F.R.C.S.Eng., Lecturer on Insanity, Westminster Hospital; Flower House, Catford, S.E.

1877. Merson, John, M.D.Aber., Medical Superintendent, Borough Asylum, Hull.

1871. Mickle, William Julius, M.D., F.R.C.P.Lond., Medical Superintendent, Grove Hall Asylum, Bow, London. (President, 1896.)

1893. Middlemass, James, M.D., F.R.C.P., C.M., B.Sc.Edin., Borough Asylum, Ryhope, Sunderland.

1898. Middlemist, George Edıyn, M.B., Moretonhampstead, Devon.

1883. Miles, George E., M.R.C.P., \&c., Medical Superintendent, Hospital for the Insane, Rydalmere, New South Wales.

1887. Miller, Alfred, M.B. and B.C.Dubl., Medical Superintendent, Hatton Asylum, Warwick.

1893. Mills, John, M.B., B.Ch., and Diploma in Mental Diseases, Royal University of Ireland, Assistant Medical Officer, District Asylum, Ballinasloe.

1881. Mitchell, R. B., M.D., Medical Supt., Midlothian District Asylum.

1885. Molony, John, F.R.C.P.I., St. Edmundsbury, Lucan, co. Dublin, Ireland.

1878. Moody, James M., M.R.C.S.Eng., L.R.C.P.\&L.M.Edin., Medical Superintendent, County Asylum, Cane Hill, Surrey.

1885. Moore, E. E., M.B.Dubl., M.P.C., Medical Superintendent, District Asylum, Letterkenny, Ireland.

1899. Moore, Win. D., M.D., M.Ch., Medical Superintendent, Holloway Sanatorium, Virginia Water, Surrey.

1892. Morrison, Cuthbert S., L.R.C.P. and L.R.C.S.Edin., Medical Superintendent, County and City Asylum, Burghill, Hereford.

1896. Morton, W. B., M.B., Assistant Medical Officer, Brislington House, Bristol.

1896. Mott, F. W., M.D., B.Sc., B.S., F.R.C.P.Lond., F.R.S., 25, Nottingham Place, W.; Puthologist, London County Asylums; Assistant Physician, Charing Cross Hospital.

1896. Mould, Gilbert E., M.R.C.S., L.R.C.P.Lond., The Grange, Rotherham, Yorks.

1862. Monld, George W., M.R.C.S. Eng., Medical Superintendent, Royal Lunatic Hospital, Cheadle, Manchester. (Prasidrart, 1880.) 
1897. Mould, Philip G., M.R.C.S.Eng., L.R.C.P.Lond., Assistant Medical Officer, Royal Lunatic Hospital, Cheadle, Manchester.

1897. Mumby, Bonner Harris, M.D.Aber., D.P.H.Cantab., Medical Superintendent, Borough Asylum, Portsmouth.

1901. Munn, Patrick James, M.B., C.M.Edin., Assistant Medical Officer, Three Counties Asylum, nr. Hitchin, Herts.

1893. Murdoch, James William Aitken, M.B., C.M.Glasg., Medical Superintendent, Berks County Asylum, Wallingford.

1900. Murphy, Jerome J., M.R.C.S., L.R.C.P.Lond., Banstead Asylum, Sutton, Surrey.

1878. Murray, Henry G., L.R.C.P.I., L.M., L.R.C.S.I., Assistant Medical Officer, Prestwich Asylum, Manchester.

1891. Musgrove, C. D., M.D.Edin., 8, Herbert Terrace, Penarth, S. Wales.

1880. Neil, James, M.D., M.P.C., Assistant Medical Officer, Warneford Asylum, Oxford.

1875. Newington, Alexander, M.B.Camb., M.R.C.S.Eng., Woodlands, Ticehurst.

1873. Newington, H. Huyes, F.R.C.P.Edin., M.R.C.S.Eng., Ticehurst, Sussex. (Presiderit, 1889.) (Treasurer.)

1893. Newington, John, M.B.Edin., Zoffany House, Bushey Hall Road, Bushes, Herts.

1881. Newth, A. H., M.D., Ardlin House, Haywards Heath, Sussex.

1869. Nicolson, David, C.B., M.D., C.M.Aber., M.R.C.P.Edin., F.S.A.Scot., Balgownie, Edgeborough Road, Guildford. (Praridmat, 1895.)

1899. Nixon, J. C., M.B., West Riding Asylum, Menston, nr. Leeds.

1893. Nobbs, Athelstane, M.D., C.M.Edin., 339, Queen's Road, Battersea Park, S.W.

1888. Nolan, Michael J., L.R.C.P.I., M.P.C., Medical Superintendent, District Asylum, Downpatrick.

1892. Noott, Reginald Harry, M.B., C.M.Edin., Senior Assistant Medical Officer, Broadmoor Criminal Lunatic Asylum, Crowthorne, Wokingham.

1880. Norman, Conolly, F.R.C.P.I., Medical Superintendent, Richmond District Agylum, Dublin, Ireland. (Hon. Secretary for Ireland, 1887-1894.) (PRerident, 1895.) (Editor of Journal.)

1885. Oakshott, J. A., M.D., Medical Superintendent, District Asylum, Waterford, Ireland.

1901. Ogilyy, David, B.A., B.Ch., M.D., L.M.Dub., Assistant Medical Officer, London County Asylum, Horton, nr. Epsom, Surrey.

1892. O’Marn, Francis, L.R.C.P.\&S.I., District Asylum, Ennis, Ireland.

1881. O’Meara, 'T. P., M.B., Medical Superintendent, District Asylum, Carlow, Ireland.

1886. O'Neill, E. D., L.R.C.P.I., Medical Superintendent, The Asylum, Limerick.

1868. Orange, William, M.D.Heidelb., F.R.C.P.Lond., C.B., Oakhurst, Godalming, Surrey. (PRrsidrat, 1883.)

1902. Orr, David, M.B., C.M.Edin., Pathologist, County Asylum, Prestwich, Lancs.

1899. Osburne, Cecil A. P., F.R.C.S.Edin., L.R.C.P.Edin., The Grove, Old Catton, Norwich.

1890. Oswald, Landel R., M.B., M.P.C., Physician Superintendent, Royal Asylum, Gartnavel, Glasgow.

1899. Owen, Corbet W., M.B., C.M.Edin., Bryn Eira, Llanfair P.G., Anglesey.

1902. Parker, Charles Seymour, M.R.C.S.Eng., L.R.C.P.Lond., Assistant Medical Officer, Darenth Asylum, Dartford, Kent.

1898. Parker, William Arnot, M.B., C.M., Gartloch Asylum, Gartcosh, N.B.

1899. Parsons, L. D., B.A., M.B., Ch.B., New Provincian Asylum, Nassau, Bahamas. 
1898. Pasmore, Edwin Stephen, M.D.Lond., M.R.C.P.Lond., Croydon Asylum, Warlingham, Surrey.

1901. Passmore, Wm. Edwi n, L.S.A.Lond., 2, Sylran Villas, Wocdford Green, Essex.

1899. Paton, Robert N., L.R.C.P., L.R.C.S.Edin., Medical Officer, H.M. Prison, Wormwood Scrubbe, London, W.

1899. Patrick, John, M.B., Ch.B., District Asylum, Belfast.

1892. Patterson, Arthur Edward, M.B., C.M.Aber., Senior Assistant Medical Officer, City of London Asylum, Dartford.

1899. Pearce, G. Heneage, M.R.C.S., Borough Asylum, Humberstone, Leicester.

1873. Pedler, George H., L.R.C.P.Lond., M.R.C.S.Eng., 6, Trevor Terrace, Knightsbridge, S.W.

1899. Penfold, William James, M.B., C.M.Edin., Assistant Medical Officer, City Asylum, Gosforth, Newcastle-on-Tyne.

1893. Perceval, Frank, M.R.C.S.Eng., L.R.C.P.Lond., Medical Superintendent, County Asylum, Prestwich, Manchester, Lancashire.

1878. Philipps, Sutherland Rees, M.D., C.M. Queen's Univ. Irel., F.R.G.S., 2, Berkeley Place, Cheltenham.

1875. Philipson, Sir George Hare, M.D. and M.A.Cantab., F.K.C.P.Lond., 7, Eldon Square, Newcastle-on-Tyne.

1891. Pierce, Bedford, M.D.Lond., M.R.C.P., Medical Superintendent, The Retreat, York.

1888. Pietersen, J. F. G., M.R.C.S., Ashwood House, Kingswinford, near Dudley, Stafford.

1898. Piper, Francis Parris, M.B.Lond., M.R.C.S., L.R.C.P., London County Asylum, Bexley, Kent.

1896. Planck, Charles, M.R.C.S.Eng., L.R.C.P.Lond., M.A.Camb., Assistant Medical Officer, East Sussex County Asylum, Haywards Heath.

1877. Plaxton, Joseph William, M.R.C.S., L.S.A.Eng., The Lunatic Asylum, Kingston, Jamaica.

1889. Pope, George Stevens, L.R.C.P.\&L.R.C.S.Edin., L.F.P.\&S.Glasg., Medical Superintendent, Middlesbrough Asylum, Cleveland, Yorks.

1901. Potts, George, I.R.C.P.\&L.R.C.S.Edin., Kent County Ophthalmic

Hospital, Maidstone.
1900. Powell, A. B. S., L.R.C.P. and S.Edin., Grahamston Asylum, Cape of Good Hope.

1876. Powell, Evan, M.R.C.S.Eng., L.S.A., Medical Superintendent, Borough Lunatic Asylum, Nottingham.

1891. Price, Arthur, M.R.C.S., L.S.A., M.P.C., Merriebank, Moss Lane, Aintree, Liverpool.

1875. Pringle, H. T., M.D.Glasg., Medical Superintendent, County Asylum, Bridgend, Glamorgan.

1901. Pugh, Robert, M.D.Edin., Ch.B., Claybury Asylum, Woodford Bridge, Essex.

1899. Rainsford, F. E., B.A., M.B., T.C.D., Resident Physician, Stewart Institute, Palmerston, co. Dublin.

1894. Rambaut, Daniel F., M.D.Univ. Dubl., Third Assistant Medical Officer and Pathologist, Richmond District Asylum, Dublin.

1902. Rattray, A. Mair, M.B., C.M.Edin., City Asylum, Gosforth, Newcastleon-Tyne.

1889. Raw, Nathan, M.D., M.P.C., Mill Road Infirmary, Liverpool.

1893. Rawes, William, M.B.Durh., F.R.C.S.Eng., Medical Superintendent, St. Luke's Hospital, Old Street, London, F.C.

1870. Rayner, Henry, M.D.Aberd.,M.R.C.P.Edin.,16,Queen Anne Street, London, W. (PRrsident, 1884.) (Late General Secretary.) (Editor of Journal.)

1899. Redington, John, L.R.C.P., L.R.C.S.I., A.M.O., Richmond Asylum, Dublin.

1887. Reid, William, M.D., Physician Superintendent, Royal Asylum, Aberdeen. 
1891. Renton, Robert, M.B., C.M.Edin., M.P.C., Courtburn, Coldingham, Berwickshire.

1886. Revington, George, M.D, and Stewart Scholar Univ. Dubl., M.P.C., Medical Superintendent, Central Criminal Asylum, Dundrum, Ireland.

1899. Rice, Darid, L.R.C.P., Cheddleton Asylum, nr. Leek, Staffs.

1897. Richard, Willinm J., M.A., M.B., C.M.Glasg., Medical Officer, Govan Parochial Asylum, Merryfluts, Govan.

1899. Richards, John, M.B., C.M.Edin., Leicestershire and Rutland Asylum, Leicester.

1889. Richards, Joseph Peeke, M.R.C.S., L.S.A., 6, Freeland Road, Ealing, W.

1893. Rivers, William H. Rivers, M.D.Lond., St. John's College, Cambridge University.

1871. Robertson, Alexander, M.D.Edin., 11, Woodside Crescent, Glasgow.

1887. Robertson, G. M., M.B., C.M., M.P.C., Medical Superintendent, District Asylum, Larbert, Stirling.

1895. Robertson, William Ford, M.B., C.M., 7, Hill Square, Edinburgh.

1900. Robinson, Harry A., M.B., Ch.B.Vict., Darenth Asylum, Dartford, Kent.

1876. Rogers, Edward Coulton, M.R.C.S.Eng., L.S.A., County Asylum, Fulbourn, Cambridge.

1859. Rogers, Thomas Lawes, M.D.St. And., M.R.C.P.Lond., M.R.C.S.Eng., Eastbank, Court Road, Elthain, Kent. (Presiderst, 1874.)

1895. Rolleston, Lancelot W., M.B., B.S.Durh., Senior Assistant Medical Officer, Middlesex County Asylum, Tooting, S.W.

1879. Ronaldson, J. B., L.R.C.P.Edin., Medical Officer, District Asylum, Haddington.

1879. Roots, William H., M.R.C.S., Canbury House, Kingston-on-Thames.

1899. Rorie, George Arthur, M.B., C.M., Senior Assistant Medical Officer, Dorset County Asylum, Dorchester.

1860. Rorie, James, M.D.Edin., L.R.C.S.Edin., Medical Superintendent, Royal Asylum, Dundee. (Late Hon. Secretary for Scotland.)

1888. Ross, Chisholm, M.B.Edin., M.D.Sydney, Hospital for the Insane, Kenmore, New South Wales.

1899. Rotherham, Arthur, M.B., B.C.Cantab., Horton Manor Asylum, near Epsom, Surrey.

1902. Round, John, I.R.C.P., L.R.C.S., L.F.P.S., 57, Ebrington Street, Plymouth.

1884. Rowe, E. L., L.R.C.P.Edin., Medical Superintendent, Borough Asylum, Ipswich.

1883. Rowland, E. D., M.D., C.M.Edin., The Public Hospital, Now Amsterdam, British Guiana.

1902. Rows, Richard Gundry, M.D.Lond., M.R.C.S., L.R.C.P., Pathologist, County Asylum, Lancaster.

1877. Russell, A. P., M.B.Edin., The Lawn, Lincoln.

1866. Rutherford, James, M.D.Edin., F.R.C.P.Edin., F.F.P.S.Glasgow, Physician Superintendent, Crichton Royal Institution, Dumfries. (Hon. Secretary for Scotland, 1876-86.)

1896. Rutherford, James M., M.B., C.M.Edin., Assistant Physician, Royal Edinburgh Asylum, Morningside.

1896. Rutherford, Robert Leonard, M.D., Medical Superintendent, Digby's Asylum, Exeter.

1892. Ruttledge, Victor, M.B., District Asylum, Londonderry, Ireland.

1902. Sall, Ernest Frederick, M.R.C.S.Eng., L.R.C.P.Lond., Assistant Medical Officer, West Sussex County Asylum, Chichester.

1894. Sankey, Edward H. O., M.A., M.B., B.C.Cantab., Resident Modical Licenseu, Boreatton Park Licensed House, Baschurch, Salop. 
- Sankey, R. Heurtley H., M.R.C.S.Eng., Medical Superintendent, Oxford County Asylum, Littlemore, Oxford.

1873. Savage, G. H., M.D.Lond., 3, Henrietta Street, Cavendish Square, W. (Late Editor of Journal.) (President, 1886.)

1899. Scott, Charles R., M.B., C.M.Edin., Bedwell Place, Abingdon.

1896. Scott, James, M.B., C.M.Edin., 19, Rsleigh Gurdeus, Brixton Hill, London, S.W.

1889. Scowcroft, Walter, M.R.C.S., Senior Assistant Medical Officer, Royal Lunatic Hospital, Cheadle, near Manchester.

1880. Seccombe, George, I.R.C.P.L., The Colonial Lunatic Asylum, Port of Spain, Trinidad, West Indies.

1879. Seed, William, M.B., C.M.Edin., The Poplars, 110, Waterloo Road, Ashton-on-Ribble, Preston.

1889. Sells, Charles John, L.R.C.P., M.R.C.S., L.S.A., White Hall, Guildford.

1902. Serjeant, Robert, M.R.C.S., L.R.C.P., Camberwell House Asylum, Peckham Road, S.E.

1882. Seward, W. J., M.B.Lond., M.R.C.S., Medical Superintendent, Colney Hatch Asylum, London, N.

1901. Shaw, B. Henry, M.B., B.Ch., B.A.O., R.M.I., Assistant Medical Officer, County Asylum, Stafford.

1891. Shaw, Harold B., B.A., M.B., B.B., D.P.H.Camb, Medical Superin. tendent, Isle of Wight County Asylum, Whitecroft, Newport, Isle of Wight.

1880. Shaw, James, M.D., 310, Kensington, Liverpool.

Shaw, T. Claye, M.D.Lond., F.R.C.P.Lond., 30, Harley Street, London, W.

1882. Sheldon, T. S., M.B., Medical Superintgndent, Cheshire County Asylum, Parkside, Macclesfield.

1300. Shera, J. E. P., L.R.C.P.I., Kent County Asylum, Chartham, near Canterbury.

1898. Sherrard, David John, B.A., M.B., M.Ch.Dubl., The Laurels, Hailsham, Sussex.

1877. Shuttleworth, G. E., M.D.Heidelb., M.R.C.S. and L.S.A.Eng., B.A.Lond., late Medical Superintendent, Royal Albert Asylum, Lancnster; Ancaster House, Richmond Hill, Surrey.

1899. Sibley, Reginald Oliver, M.B.Lond., M.R.C.S., L.R.C.P., Assistant Medical Officer, Loudon County Asylum, Cane hill, Purley, Surrey.

1901. Simpson, Alexander, M.A., M.D.Aber., Medical Superintendent, County Asylum, Winwick, Newton-le-Willows, Lancashire.

1895. Simpson, Francis Odell, M.R.C.S., L.R.C.P., Senior Assistant Medical Officer, County Asylum, Rainhill, near Liverpool.

1888. Sinclair, Eric, M.D., Medical Superintendent, Gladesville Anylum, New South Wales.

1891. Skeen, James Humphrey, M.B., C.M.Aber., Medical Superintendent, Glasgow District Asylum, Bothwell.

1898. Skeen, William St. John, M.B., C.M., County Asylum, Winterton, Ferryhill, Durham.

1900. Skinner, Eruest W., M.D., C.M.Edin., Bank House, Rye, Sussex.

1901. Slater, G. N. O., M.D., Assistant Medical Ofincer, Essex County Asylum, Brentwood.

1897. Smalley, Herbert, M.D.Durh., L.R.C.P., M.R.C.S., Prison Commission, Home Office, Whitehall, S.W.

1899. Smith, J. G., M.D., Herts County Asylum, Hill End, St. Albans, Herts.

1885. Smith, R. Percy, M.D., B.S., F.R.C.P., M.P.C., 36, Queen Anne Street, Cavendish Square, W. (General Secretary, 1896-7.)

1858. Smith, Robert, M.D.Aber., L.R.C.S.Edin., Middelton Hall, Middelton St. George, Durhrm.

1884. Smith, W. Beattie, F.R.C.S.Edin., L.R.C.P.Lond., Medical Superintendent, Hospital for the Insane, Kew, Melbourne, Victoria.

1901. Smyth, R. B., M.D., Ch.B., Senior Assistant Medical Oficer, County Asylum, Gloucester. 
1899. Smyth, Walter, M.B., B.Ch., R.U.I., Assistant Medical Oficer, County Asylum, Antrim.

1881. Snell, George, M.D.Aber., M.R.C.S.Eng., Vine Cottage, Norwood Green, Southall, Middlesex.

1885. Soutar, James G., M.B., Barnwood House, Gloucester.

1883. Spence, John Buchan, M.D., M.C., The Asylum, Colombo, Ceylon.

1875. Spence, J. Beveridge, M.D., M.C.Queen's Uuiv., Medical Superintendent, Burntwood Asylum, near Lichtield. (Presiderst, 1899-1900, formerly Registrar.)

1899. Spicer, A. H., M.B., B.S.Lond., Petworth, Sussex.

1898. Sproat, James Hugh, M.B.Lond., M.R.C.S., L.R.C.P., Somerset and Bath Asylum, Wells.

1891. Stansfield, T. k. K., M.B., C.M.Edin., The Heath Asylum Bexley, Kent.

1901. Starkey, William, M.B., B.Ch., B.A.O.Roy. Univ. Irel., Assistant Medical Officer, Lancashire County Asylum, Prestwich, near Manchester.

1898. Steen, Robert H., M.D.Lond., West Sussex Asylum, near Chichester.

1899. Stevens, Reginald C. J., M.B., B.S.Durh., County Asylum, Exminster, Devon.

1868. Stewart, James, B.A.Queen's Univ.Irel., F.R.C.P.Edin., L.R.C.S.Irel., late Assistant Medical Officer, Kent County Asylum, Maidstone; Dunmurry, Sneyd Park, near Clifton, Gloucestershire.

1884. Stewart, Robert S., M.D., C.M., Assistunt Medical Officer, Angelton Bridgend, Glamorgan.

1887. Stewart, Rothsay C., M.R.C.S., Medical Superintendent, County Asylum, Leicester.

1862. Stilwell, Henry, M.D.Ediu., M.R.C.S.Eng., Moorcroft House, Hillingdon, Middlesex.

1899. Stilwell, Reginald J., M.R.C.S., L.R.C.P., Moorcroft House, Hillingdon, Middlesex.

1864. Stocker, Alonzo Henry, M.D.St. And., M.R.C.P.Lond., M.R.C.S.Eng., Medical Superintendent, Peckham House Asylum, Peckham.

1897. Stoddart, William Henry Butter, M.D., B.S.Lond., M.R.C.S.Eng., M.R.C.P.Lond., Bethlem Royal Hospital, London, S.E.

1900. Stracey, Bernard, M.B., Ch.B.Edin., Sutton-Bonnington, Loughborough

1885. Street, C. T., M.R.C.S., L.R.C.P., Haydock Lodge, Ashton, Newton-leWillows, Lancashire.

1900. Stuart, Esther Molyneux, M.B., C.M.Edin., County Asylum, Morpeth, Northumberland.

1900. Stuart, F. J., M.R.C.S., L.R.C.P., Berrywood Asylum, Northampton.

1897. Stuart, Robert, M.R.C.S., L.R.C.P.Lond., 20, New Elvet, Durham.

1900. Sturrock, James Pain, M.A., M.B., C.M.Edin., Midlothian and Peebles Asylum, Rosslynlee, N.B.

1886. Suffern, A. C., M.D., Medical Superintendent, Ruberry Hill Asylum, near Bromsgrove, Worcestershire.

1894. Sullivan, W. C., M.D.R.U.I., H.M. Prison, Pentonville, London, N.

1898. Sutcliffe, John, M.R.C.S., L.R.C.P., Royal Asylum, Cheadle, near Manchester.

1895. Sutherland, John Francis, M.D.Edin., Deputy Commissioner in Lunacy, 19, Mayfield Road, Edinburgh.

1877. Swanson, George J., M.D.Edin., The Pleasaunce, Heworth Moor, York.

1901. Sykes, Arthur, M.R.C.S., L.R.C.P., Assistant Medical Officer, City Asylum, Hellesdon, nr. Norwich.

1897. Tait, James Sinclair, M.D., L.R.C.P.Lond., F.R.C.S.Edin., L.R.C.P. Edin., D.P.H.Edin., R.C.P.S.Edin., F.P.S.Glasg., Medical Superintendent, Hospital for Insane, St. John's, Newfoundland. 
1857. Tate, William Barney, M.D.Aber., M.R.C.P.Lond., M.R.C.S.Eng., Medical Superintendent of the Lunatic Hospital, The Coppice, Nottingham.

1897. Taylor, Frederic Ryott Percival, M.D., B.S.Lond., M.R.C.S.Eng., L.R.C.P.Lond., Darenth Asylum, Dartford, Kent.

1890. Telford-Smith, Telford, M.A., M.D., Wimborne, Dorset.

1888. Thomas, E. G., Haveringwell, Caterham, Surrey.

1880. Thomson, D. G., M.D., C.M., Medical Superintendent, County Asylum, Thorpe, Norfolk.

1902. Thomson, Eric M., M.A., M.B., Ch.B., James Murray's Royal Asylum, Perth, N.B.

1902. Thomson, James, M.D., Gartloch Hospital for Mental Diseases, Gartcosh, N.B.

1901. Tighe, John, M.B., B.Ch., B.A.O.Irel., North Riding Asylum, Clifton, Yorks.

1900. Tinker, William, L.R.C.P., Holloway Sanatorium, Virginia Water, Surrey.

1898. Todd, Percy Everald, M.B., Medical Superintendent, Pretoria Asylum, Transvaal, South Africa.

1901. Torney, George Parsons, A.B.Dubl., L.R.C.P., L.R.C.S.I., L.M., Medical Superintendent, County Asylum, Lincoln

1896. Townsend, Arthur A. P., M.R.C.S.Eng., L.R.C.P.Lond., Aseistant Medical Officer, Hospital for Insane, Barnwood House, Gloucester.

1902. Trevelyan, Edmund Fauriel, M.D.Lond., F.R.C.P.Iond., Assistant Physician to the Leeds General Infirmary, 40, Park Square, Leeds.

1881. Tuke, Charles Molesworth, M.R.C.S.E., Chiswick House, Chiswick.

1888. Tuke, John Batty, jun., M.B., C.M., M.R.C.P.E., Resident Physician, Saughton Hall, Edinburgh.

1885. Tuke, T. Seymour, M.B., B.Ch.Oxford, M.R.C.S.E., Chiswick House, Chiswick, W.

1877. Turnbull, Adam Robert, M.B., C.M.Edin., Medical Superintendent, Fife and Kinross District Asylum, Cupar. (Hon. Secretary for Scotland.)

1889. Turner, Alfred, M.D., C.M., Plympton House, Plympton, S. Devon.

1890. Turner, John, M.B., C.M.Aberd., Senior Assistant Medical Officer, Essex County Asylum, Brentwood.

1878. Urquhart, Alex. Reid, M.D., F.R.C.P.E., Physician Superintendent, James Murray's Royal Asylum, Perth. (Editor of Jourmal.) (Hos. Secretary for Scotland, 1886-94.) (PReBIDEkT, 1898.)

1900. Veitch, J. Ogilvie, M.B., C.M.Edin., County Asylum, Powick, Worcester.

1894. Vincent, William James, M.B.Durh., Assistant Medical Officer, Wadeley Asylum, near Sheffield.

1884. Walker, E. B. C., M.B., C.M.Edin., Assistant Medical Officer, County Asylum, Haywards Heath.

1896. Walker, William F., L.R.C.S.\&L.M.Edin., L.S.A.Lond., Plas-yn-Dinas, Diuas Mawddwy, Merionethshire.

1898. Wull, Charles Percivale Bligh, M.B., Ch.B.Edin., Butterworth, Transkei, Cape Colony.

1877. Wallace, James, M.D., Visiting Medical Officer, 16, Union Street, Greenock.

1900. Walters, John Basil, M.R.C.S.Eng., L.R.C.P.Lond., Kingsdown House, Box, Wilts.

1889. Warnock, John, M.D., C.M., B.Sc., Abassia, Egypt.

1895. Waterston, Jane Elizabeth, M.D.Bru., L.R.C.P.I., L.R.C.S.Edin., 53, Parliament Street, Cape Town, South Africa.

1902. Watson, Frederick, M.B., C.M.Edin., Assistant Medical Officer, Ayr District Asylum, Ayr, N.B.

1891 Wateon, George A., M.B., C.M.Edin., M.P.C., 29, Abbot's Park Road, Leyton, Essex. 
1885 Watson, William Riddell, L.R.C.S. and L.R.C.P.Edin., Govan District Asylum, Hawkhead, Paisley.

1898. Watson, William R. K., M.A., M.B., C.M., 18, Montrell Road, Streatham Hill, London, S.W.

1880. Weatherly, Lionel A., M.D., Bailbrook House, Bath.

1902. Welch, Frederick Day, M.R.C.S., L.R.C.P.Lond., Assistant Medical Officer, Burghill Asylum, Hereford.

1897. Welsh, Gilbert Aitken, M.B., C.M.Edin., The Crescent, Garliestown, N.B

1880. West, George Francis, L.R.C.P.Edin., Medical Superintendent, District Asylum, Kilkenny, Ireland.

1872. Whitcombe, Edmund Banks, M.R.C.S., Medical Superintendent, Winson Green Asylum, Birmingham. (Presiderst, 1891.)

1884. White, Ernest William, M.B.Lond., M.R.C.P.Lond., Resident Physiciun and Superintendent, City of London Asylum, nr. Dartford, Kent. (Hon. Sec. South Eastern Division, 1897-1900.)

1889. Whitwell, James Richard, M.D. and C.M., Medical Superintendent, Suffolk County Asylum, Melton Woodbridge.

1883. Wiglesworth, J., M.D.Lond., Rainhill Asylum, Lancashire.

1895. Wilcox, Arthur William, M.B., C.M.Edin., Second Assistant Medical Ofticer, County Asylum, Hatton, Warwick.

1900. Wilkinson, H. B., M.R.C.S., L.R.C.P., Assistant Medical Officer, Plymouth Borough Asylum, Blackadon, Ivybridge, South Devon.

1887. Will, John Kennedy, M.B., C.M., M.P.C., Bethnal House, Cambridge Road, N.E.

1902. Willis, Wm. Frederick, M.R.C.S.Eng., L.R.C.P.Lond., Assistant Medical Officer, County Asylum, Exminster, Devon.

1901. Wilson, Albert, M.D.Edin., Minto House, South Woodford, Essex.

1890. Wilson, George R., M.B., C.M., M.P.C., Medical Superintendent, Linden Lodge, Loanheak.

1900. Wilson, Jumes Patterson, M.B., Ch.B.Glasg.

1896. Wileon, Robert, M.B., C.M.Glasg., Nailsworth, Gloucestershire.

1897. Winder, W. H., M.R.C.S., L.R.C.P.Lond., D.P.H.Cantab., Deputy Medical Officer, H.M. Convict Prison, Aylesbury.

1875. Winslow, Henry Forbes, M.D.Lond., M.R.C.P.Lond., 14, York Place, Portman Square, London.

1897. Wiseman, David William, M.R.C.S.Eng., L.R.C.P.Lond., 300, Commercial Road, Portsmouth.

1894. Wood, Guy Mills, M.B.Durh., 6, Woburn Square, London, W.C.

1869. Wood, T. Outterson, M.D., M.R.C.P.Lond., F.R.C.P., F.R.C.S.Edin. 40, Margaret Street, Cavendish Square, W.

1885. Woods, J. F., M.R.C.S., Medical Superintendent, Hoxton House, N.

1873. Woods, Oscar T., M.B., M.D.Dubl., L.R.C.S.I., Medical Superintendent, District Asylum, Cork. (Hon. Secretary for Ireland, 1897.) (РRBSIDENT, 1901.)

1900. Worth, Reginald, M.R.C.S., L.R.C.P., Middlesex County Asylum, Wandsworth, S.W.

1877. Worthington, Thomas Blair, M.A., M.B., and M.C.Trin. Coll., Dubl., Medical Supt., County Asylum, Knowle, Fareham, Hants.

1898. Yeates, Thomas, M.B., C.M., Borough Asylum, Ryhope, Sunderland.

1862. Yellowlees, David, M.D.Edin., F.F.P.S.Glasg., LL.D., 6, Albert Gate, Dowan Hill, Glasgow. (Prrsident, 1890.) 
xxiv

Members of the Association.

\begin{tabular}{rrrrrrr} 
ORDINARY MRMBRRs & $\ldots$ & $\ldots$ & $\ldots$ & $\ldots$ & $\ldots$ & 586 \\
$\begin{array}{l}\text { HONORARY M MMBRRS } \\
\text { CoRREBPONDING MEMBERS }\end{array}$ & $\ldots$ & $\ldots$ & $\ldots$ & $\ldots$ & 37 \\
& & & $\ldots$ & $\ldots$ & $\ldots$ & 12 \\
\hline Total & $\ldots$ & $\ldots$ & $\ldots$ & $\ldots$ & $\mathbf{6 3 5}$
\end{tabular}

Members are particularly requested to send changes of address, \&c., to Dr.

Rolert Jones, the Honorary Secretary, 11, Chandos Street, Cavendish

Square, London, $W$., and in duplicate to the Printers of the Journal,

Messrs. Adlard and Son, $22 !$ Bartholomevo Close, London, E.C. 
List of those who have passed the Examination for the Certificate of Efficiency in Paychological Medicine, entitling them to append M.P.C. (Med. Psych. Certif.) to their names.

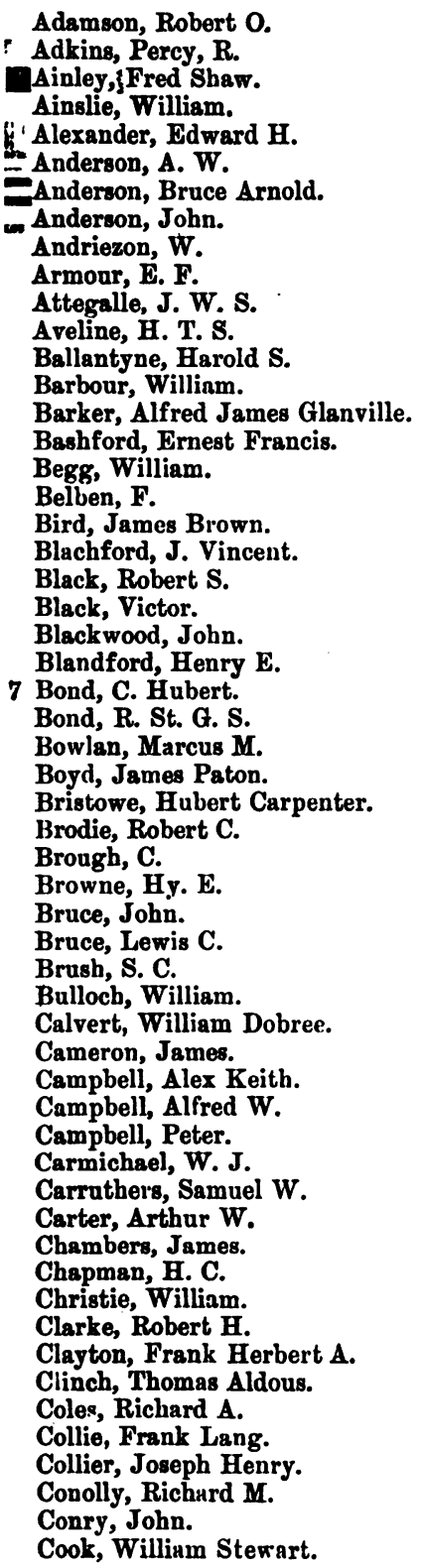

Cooper, Alfred J. S.

Cope, George Patrick.

Corner, Harry.

Cotton, William.

Couper, Sinclair.

Cowan, John J.

Cowie, C. G.

Cowie, George.

Cowper, John.

Cox, Walter $\mathbf{H}$.

8 Craig, $M$.

Cram, John.

Crills, G. H.

Cross, Edward John.

Cruickshank, George.

Cullen, George M.

Cunningham, James F.

Dalgetty, Arthur B.

Davidson, Andrew.

Davidson, William.

6 Dawson, W. R.

De Silva, W. H.

Distin, Howard.

Donald, 'Wm. D. D.

Donaldson, R. L. S.

Donellan, James O'Conor.

Douglas, A. R.

Downey, Augustine.

Drummond, Russell J.

Eames, Henry Martyn.

Earls, James H.

East, W. Norwood.

Easterbrook, Charles C.

Eden, Richard A. S.

Edgerley, S.

Edwards, Alex. H.

Elkins, Frank A.

Ellis, Clarence J.

English, Edgar.

Eustace, J. N.

Eustace, Henry Marcus.

Evans, P. C.

Ewan, John A.

Ezard, Ed. W.

Falconer, James F.

Farquharson, Wm. Fredk.

Fennings, A. A.

Ferguson, Robert.

Findlay, G. Landsborough.

Fitzgerald, Gerald.

Fleck, David.

Fox, F. G. T.

Fraser, Donald Allan.

Fraser, Thomas.

Frederick, Herbert John.

Gaudin, Francis Neel. 
xxvi

Gawn, Eruest K.

Geminall, William.

Genney, Fred. $\mathbf{8}$.

Gibeon, Thomas.

Giles, $\mathbf{\Lambda}$ : B.

Gill, J. Macdonald.

Gilmour, John R.

Goldie, E. M.

Goldschmidt, Oscar Bernard.

Goodall, Edwin.

Graham, Dd. James.

Graham, F. B.

Grainger, Thomas.

Grant, J. Wemyes.

Grant, Lacklan.

Gray, Alex. C. E

Griffiths, Edward $\mathbf{H}$.

Hall, Harry Baker.

Halsted, H. C.

Haalam, W. A.

Haslett, William John Handfield.

Hassell, Gray.

Hector, William.

Henderson, Jane B.

Henderson, P. J.

Hennan, George.

Hewat, Matthew $\mathbf{L}$.

Hicks, John A., jun.

Hitchings, Robert.

Holmes, William.

Horton, James Henry.

Hotchkis, R. D.

Howden, Robert.

Hughes, Robert.

Hutchinson, P. J.

2 Hyslop, Thos. B.

Ingram, Peter $\mathbf{R}$.

Jagannadhan, Annie W.

Johnston, John M.

Kelly, Francis.

Kelso, Alexander.

Kelson, W. H.

Ker, Clande B.

Kerr, Alexander L.

Kejt, Frederick.

King, David Barty.

King, Frederick Truby

I Laing, C. A. Barclay.

Laing, J. H. W.

Law, Thomas Bryden.

Leoper, Richard $\mathbf{R}$.

Leslie, R. Murray.

Liveen, Arthur W. Bligh.

Livingatone, John.

Lloyd, R. H.

Low, Alexander.

McAllum, Stewart.

Maedonald, David.

Macdonald, G. B. Douglas.

Macdonald, John.

Maceroy, Henry John.

McGregor, George.
Macinnes, Ian Lamont.

Mackenzie, Henry J.

Mackenzie, John Cuinming.

Mackenxie, William H.

Mackensie, William $\mathbf{L}$.

Mackie, Cteorge.

MCLean, H. J.

Mucwillan, John.

5 Macnanghton, Geo. W. F.

Macneice, J. G.

Macpherson, John.

Macvean, Donald A.

Mallannah, Sreenagula.

Marr, Hamilton C.

Marsh, Ernest $L$.

Martin, A. A.

Martin, A. J.

Martin, Wm. Jewis.

Masson, James.

Meikle, T. Gordon.

Melville, Henry B.

Middlemass, James.

Mitchell, Alexander.

Mitchell, Charles.

Moffett, Flizabeth J.

Monteith, James.

Moore, Edwrard Errkine.

1 Mortimer, John Desmond kirnest.

Murison, Cecil C.

Myers, J. W.

Nair, Charles R.

Nairn, Robert.

Neil, James.

Nixon, John Clarke.

Nolan, Michael James.

Norton, Everitt E.

Orr, David.

Orr, James.

Orr, J. Fraser.

Oswald, Landel $R$.

Paget, A. J. M.

Parker, William A.

Parry, Charles P.

Patterson, Arthur Edward.

Putton, Walter S.

Paul, William Moncrief.

Pearce, Walter.

Penfold, William James.

Philip, James Farquhar.

Philip, William Marzhall.

Pieris, William C.

Pilkington, Frederick $W$.

Pitcairn, John James.

Porter, Charlea.

Price, Arthur.

Pring, Horace Reginald.

Rainy, Harry, M.A.

Ralph, Richard M.

Rannie, James.

4 Raw, Nathan.

Reid, Matthew 1.

Rentou, Robert. 


\section{zxvii}

Rice, P. J.

Rigden, Alan.

Ritchie, Thomas Morton.

Rivers, W. H. R.

3 Robertson, G.M.

Robson, Francis Wm. Hope.

Rorie, George A.

Rose, Andrew.

Rowand, Andrew.

Rudall, James Ferdinand.

Rust, James.

Rust, Montague.

Rutherford, J. M.

Sawyer, Jas. E. H.

Scott, George Brebner.

Scott, J. Walter.

Scott, William T.

Sheen, Alfred W.

Simpson, John.

Simpson, Samuel.

Skme, F. M. T.

Skeen, George.

Skeen, James H.

Slater, William Arnison.

Smith, Percy.

Smyth, William Johnson.

Snowball, Thomas.

Soutar, James G.

Sproat, J. H.

Stanley, John Douglas.

Staveley, William Henry Charles.

Steel, John.

Stephen, George.

Stewart, William Day.

Stoddart, John.
9 Stoddart, William H5. B.

Strangman, Lucia.

Strong, D. R. T.

Stuart, William James.

Symes, G. D.

Thompson, George Matthew.

Thomson, Eric.

Thomson, George Felix.

Thorpe, Arnold $\mathrm{E}$.

Trotter, Robert Samuel.

Turner, W. A.

Umney, W. F.

Walker, James.

Warde, Wilfred B.

Waterston, Jane Elizabeth.

Watson, George $\mathbf{A}$.

Welsh, Darid A.

West, J. T.

Whitwell, Robert R. H.

Wickham, Gilbert Henry.

Will, John Kennedy.

Williams, D. J.

Williamson, A. Maxwell.

4 Wilson, G. R.

Wilson, Jumes.

Wilson, John T.

Wilson, Robert.

Wood, David James.

Wright, slexander, W. 0.

Yeates, Thomas.

Yeoman, John B.

Young, D. P.

Younger, Henry J.

Zimmer, Carl kaymond.

1 To whom the Gaskell Prize (1887) was awarded. 2 To whom the Gaskell Prize (1889) was awarded.

3 To whom the Gaskell Prize (1890) was awarded.

4 To whom the Gaskell Prize (1892) was awarded.

5 To whom the Gaskell Prize (1895) was awarded.

6 To whom the Gaskell Prize (1896) was a warded.

7 To whom the Gaskell Prize (1897) was awarded.

8 To whom the Gaskell Prize (1900) was awarded.

9 To whom the Gaskell Prize (1901) was awarded. 
JOURNAL OF MENTAL SCIENCE, JANUARY, I 903.

Male Nurses' (TEMPERANGE) Co-operation, Only. rLondon: 10, THAYER STREET, MANCHESTER SQUARE, W. Addresses - ¿ Manchester: 235, BRUNBWICK 8T. (Facing Owens College).

Superior Trained MALE NURSES for Medical, Surgical, Mental, Dipsomania, Fever, anil Travelling Cases supplied at a moment's notice, Day or Night. All Nurses supplied by this Association are TOTAL ABSTAINERS. SKILLED MASSEURS supplied. Terms: $f_{1} 11 s$. 6d. to $f_{2}$ 28. or upwards per week. Nurses to receive their own fees. F. Rorst, Secretary.

Telephones $\left\{\begin{array}{c}\text { (London), 539, Paddington. } \\ (\text { MInchester), 4699, Central. }\end{array}\right.$ Telegrams $\left\{\begin{array}{l}\text { “Assuaged, London." } \\ \text { "Assuaged, Mfanchrster." }\end{array}\right.$

CAUTION.-Certain persons having no connection with this Association are copying our Business Cards and Advertisements, adding a different Address.

\section{$\mathbf{R} \mathbf{E}{ }^{\text {ALwars }} \mathbf{L} \mathbf{L} \mathbf{E} \mathbf{X}$}

FLOOR POLISH.

REQUIRES NO MIXING WHATEVER.

Brilliantly Polishes Floors, Linoleums, Floorcloths, \&c.

Economical, Cleanly, Healthful; Maximum of Lustre for Minimum of Labour.

GE0. T. COX \& SONS, Ltd., 35, EASTCHEAP, E.C.

This Polish is specially adapted for use in Public Institutions and other large establishments. Samples Free on application.

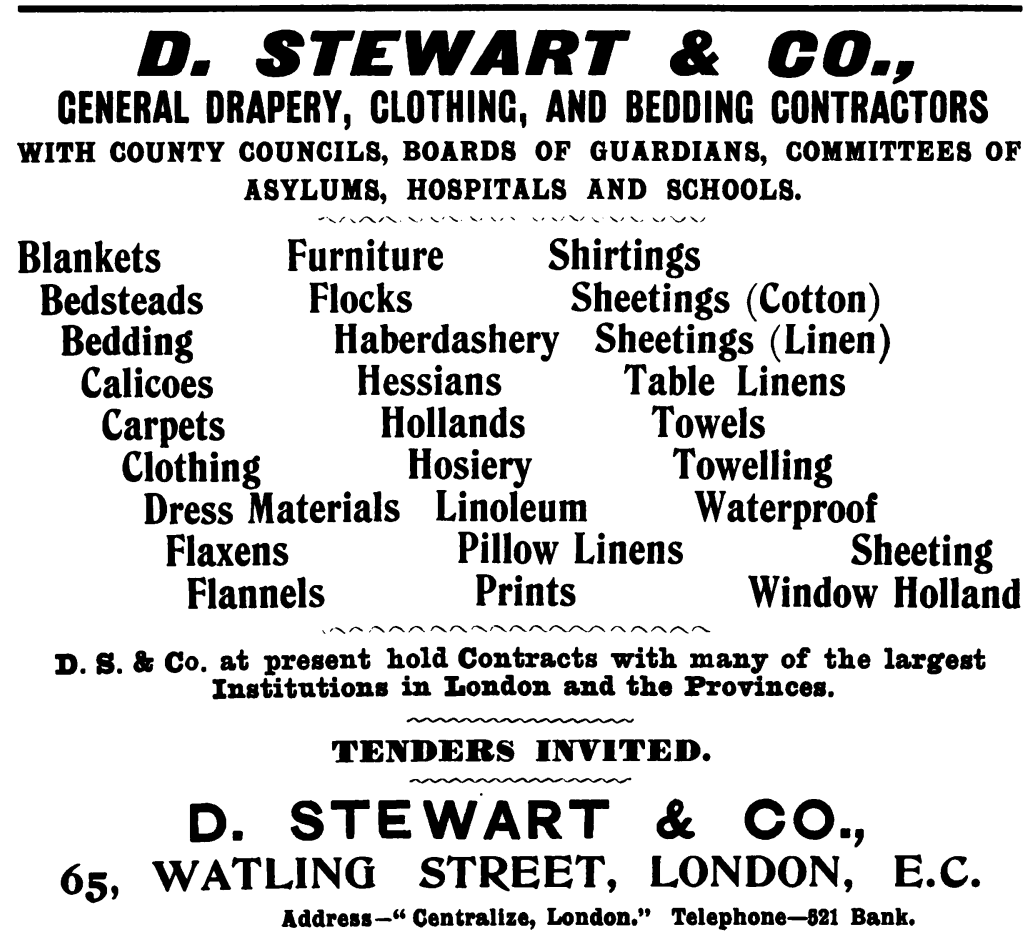


15, Gt. Marylebone Street, Portland Place, London, W. ;

\section{6, Oxford Street. Manchester.}

Incorporated under the Industrial and Provident Societies Act, 1893. (Regd. No. 3685.)

Highly trained Male Nurses for Mental, Medical, Surgical, Catheter, and all cases. Terms from \&1 11s. 6d. upwards. Masseurs and Valet Attendants also supplied.

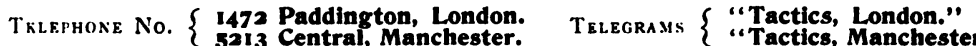
M. D. GOLD, Sec., London. W. BLADES, Sec., Manchester.

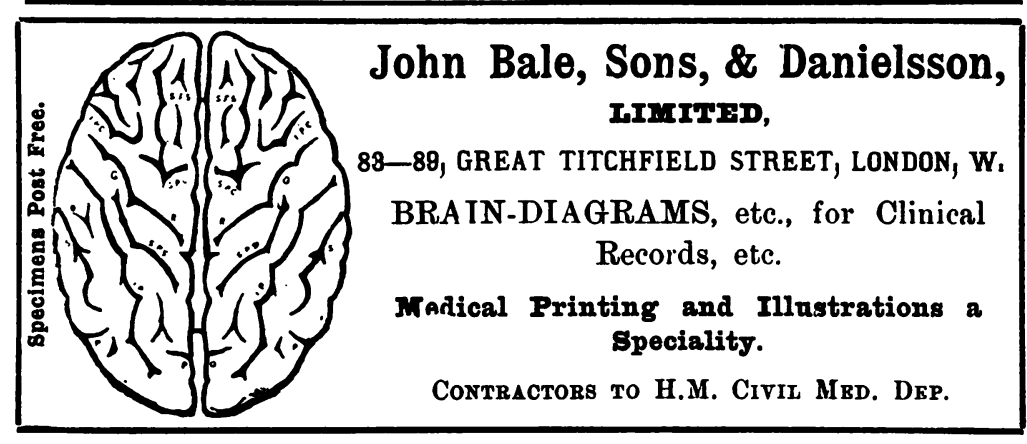

TREATMENT OF INEBRIATES-GentLEFOLK ONLY.-DR. STEWART, of "Dunmurry," Sneyd Park, near Clifton (a teetotal home, Estd. 1876), and Mrs. S. receire as voluntary boarders in thei: family three Ladies and three Gentlemen of guod social position anxious to be permanently cured of inebriety. Refined society. Bracing air. No "nersous" cases received. No legal formalities.

Telegrams : “ Dr. STFWART, Dunmurry, Rocklenze, Bristol."

\section{H.R.H. PRINCESS CHRISTIAN'S TRAINED NURSES.}

Fully (Three years) Hospital Trained Nurses for Medical, Surgical, Infectious, Mental, Maternity, and Massage Cases

Can be supplited on the Shortest Notioe.

APPLY BY TELegram OR OTHERWISE TO

The Superintendent, 1, CLARENCE YILLAS, WINDSOR. Telephone-

OI57, WINDSOR.

Telegraphic Address"NURSES, Windsor."

All business communications relating to Advertisements to be addressed to Messi'8. J. H. Booty \& Son, 30, Holborn, E.C. 
JOURNAI, OF MENTAI, SCIENCE, JANUARY, I9O3. iii

Telegrams: "ATKINSON COMPANY, LONDON." Telephone: 700 HOP.

\section{ATKINSON \& CO., \\ GOVRRTMETT CONTRACrORE,}

$198,200,202,204,206,208,210,212$, WESTMINSTER BRIDGE ROAD, LONDON,

Have the honour to hold some of the largest Contracts in the Kingdom from Asylums, Unions, Hospitals, and Government Departments. The magnitude of their Contracts enables them to employ Manufacturers solely for their demands, thus affording a direct charge to the Consumer.

NUMBROUS SPECIALITIES IN

HOSPITAL BEDSTEADS, CHAIRS, SEATS, MATTRESSES, LOCKERS, \&c. \&c.

NAPIER MATTING, LINOLEUMS, AND VARIOUS FLOOR COVERINGS

Made in Special Widths and Qualities to meet exceptional wear.

" ATKINSON'S" PINE FIBRE MATTRESS is specially recommended because of its healthful and antiseptic properties.

PRICES ON APPLICATION.

ATKINSON \& CO. undertake to supply any quantity of Goods as specified below by the quarter, half-yeur, or year, viz.:

Bedsteads in Wood or Iron, Iron Bedsteads with Patent Spring Mesh Bottoms, Woren Wire and other Mattresses, Coir, Fibre,

Horsehair, Flocks, and every other Material for Bedding.

Blankets, Rugs, Counterpanes.

Shoeting, Waterproof ditto.

Flannels, Calicoes, Prints, Woollen Cloths of all qualities. Corduroys, Fustians, \&c.

Men and Women's Under and Upper Clothing.

Ready-made Garments, in all sizes and for both sexes. Hats, Caps, Bonnets, \&c.

Fosiery and Haberdashery. Uniforms, \&c.

FURNITURE, CARPETS, and every Requisite supplied on the Shortest Notice for Board Rooms, Offices, Masters' Houses, \&c.

ILlustrated CATAlogues aNd estimates FREE.

Secretaries and Clerks of Institutions are respectfully requested, when issuing Contracts, to forward a Form of Tender to Atrinson \& Co.

SPFCIAL CATALOGUE POST FRFE ON APPLICATION. 


\title{
THE THYROID TREATMENT and the Preparations manufactured by ALLEN \& HANBURYS LTD.

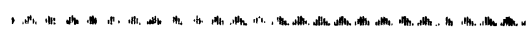

Myxodema.-The relief afforded to cases of Myxodema by the use of the Thyroid Gland during the last five years has marked a totally new departure in the history of medicine. We are beginning to understand the physiological importance of Internal Secretions to the animal economy. Myxodema has been recognised as long ago as 1874 by Sir Wm. Gull as a distinct disease, and
hefore that time it lind been found that nnimals invariably succumbed afier total extirpation of the Thyroid.

Cretinism, too, had long heen recognised to be connected with congenital absence or deficiency in the glandular structure of the Thyroid.

Time enough has now elapsed to prove that those cases of Myxoedema which have been restored to health maintain that condition as long as they continue to take the gland or efficient preparation of its extrict.

Sporadic Cretinism.-As regards Sporadic Cretinism, we cannot do better than refer the reader to an original article which appeared in the Lancet, with Photographs, by W. W. $0-$ M.D. Oxon., M.R.C.P. London, November 4 th, 1893, p. 1113 .

All these cases were treated by Allen and Hanburys' Thyroidin Tablets. The results are perhaps more remarkable than those obtained in the allied condition of Myxodema. The writer observes that these Preparations have given entire satisfaction. The Photographs speak for themselves.

The success attending the use of Thyroid Extract in the Myxœdematous condition of the skin and its appenduges has suggested its employment in various skin lesions.

Psoriasis. - Vory good results have been attained in some cases of obstinate psoriasis by using Thyroidin Tablets.

In a recent number of the Lancet three of these cases are reported. After treatment with iodide of potassium and arsenic internally, and clirysophanic acid ointment lochlly, sliowed no improvement, but, on the other hand. the disorder was in some cases aggravated, developing a general dermatitis of the face with febrile disturbance.

But on the treatment being changed, and TABLrTs of Thyroid GLAND (AlLRN AND HaNBUrys') being administered, the writer observes:

Case 1.- "The inprovement was very striking, ... the scales were completely gone, and all that was left was a slight discoloration of the skin where the disease had appeared."

Case 2.-"The scrlp and limbs were nearly clear. . . The patient, who has suffered from the disease for nineteell years past, has had no discomfort from the remedy, and says that notling has ever acted so rapidly upon his disease."

Case 3.- "The result was astonishing, for in three days there was a marked improvement, and no further spread of the disease had taken place. No discomfort was experienced from the remedy.

For full details of the above quotations see the Lancet, January 6th, 1894, p. 19

Whilst formerly liquid extracts aud the raw gland were used, Al.LKN AN D HANBURYs were the first to offer the active principles of the Thyroid Gland in the compressed form, which is now the favourite mode of administration. The glands are dissected and carefully exumined and separated from all blood fat, and inert tissue, which readily decompose and produce gastric disturbances, romiting, \&c., and which give to sume preparations on the market a most repulsive smell, especially when they have been kept for a short time.

\section{THYROIDIN TABLETS}

Rexdily disintegrate when 3 wallowed, and from their shape and size are taken without difficulty. If preferred, they may be dissolved in a little water before being swallowed.

THYROIDIN CACHETS

Contain 5 grs. of Thyroidin in each. Dose : 1 or 2 . A rery suitable and convenient mode of taking Thyroidin.

\section{ELIXIR THYROIDIN} Is a very palatable, slightly sweet preparation, of whlich one fluid ounce represents one Gland (average).
It will be found to keep well without cliange. Dose : 1 to 4 fluid drachms.

The above are put up in 18., 2s. 6d., and 4s. 6d. packages (retail), or 8s., 218., and 40s. per doz. to the Profession.

SAMPLES SUPPLIED TO THE PROFESSION ON APPLICATION.

\author{
ALLEN \& HANBURYS LTD., \\ Plough Court, Lombard Street, LONDON.
}




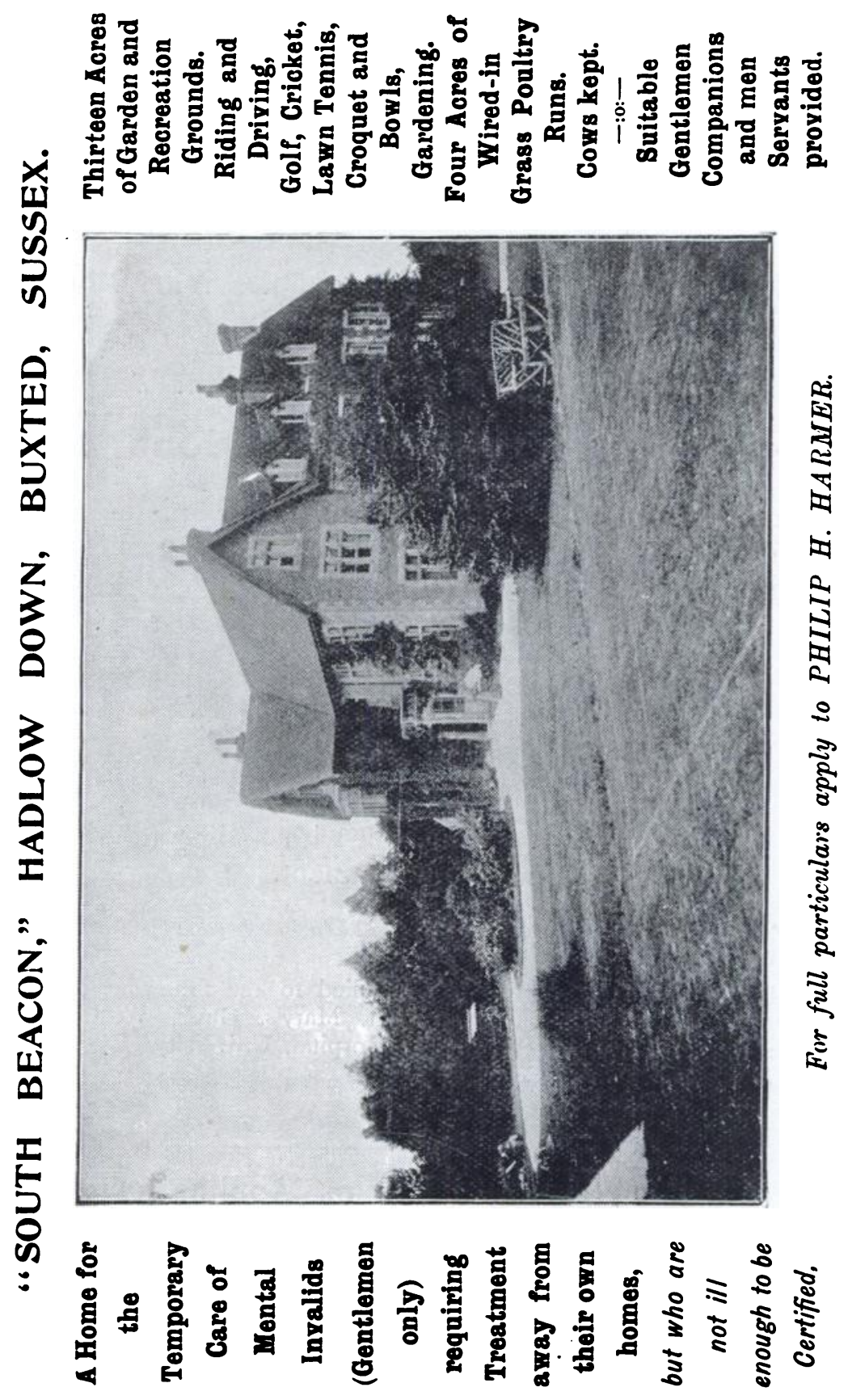


vi JOURNAL OF MENTAL SCIENCE, JANUARY, 1903.

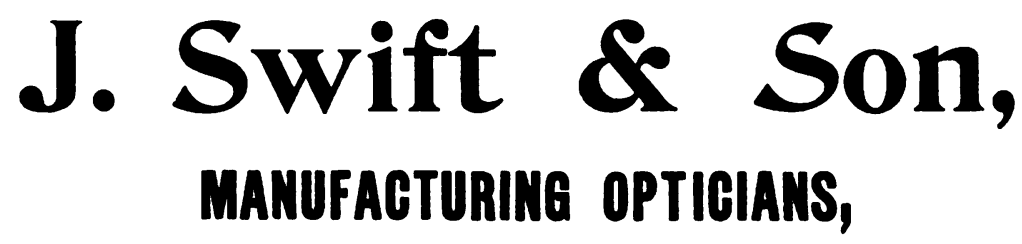

Solely appointed to H.M. Dept., War Office.

\section{NEW HISTOLOGICAL MICROSGOPE,}

with coarse adjustment by rack and pinion, fine adjustment by micrometer screw, $2 / 3^{\prime \prime}$ and $1 / 6^{\prime \prime}$ objectives, one ocular and iris diaphragm, in Cabinet.

\section{16s.}

Double nose-piece as figured (extra).

\section{8.}

Microscope as above, but with sliding tube, coarse adjustment.

\section{5s.}

These nicroscopes have been supplied to the following medical schools, viz.-St. Bartholomew's, St. Mary's, Guy's, Westminster, Charing Cross, Middlesex, London Hospital, University College, and most of the provincial Colleges.

Illustrated Circular on Application.

\section{University Optical Works,}

81, TOTTEHHAM GOURT ROAD, W. 